\title{
Loss of Wasl improves pancreatic cancer outcome
}

\author{
Ana Hidalgo-Sastre, ${ }^{1}$ Judit Desztics, ${ }^{1}$ Zahra Dantes, ${ }^{1}$ Katharina Schulte, ${ }^{1}$ Hilal Kabadayi Ensarioglu, ${ }^{1,2}$ \\ Blessing Bassey-Archibong, ${ }^{3}$ Rupert Öllinger, ${ }^{1,4}$ Thomas Engleiter, ${ }^{1,4}$ Lyndsay Rayner, ${ }^{3}$ \\ Henrik Einwächter, ${ }^{1}$ Juliet M. Daniel, ${ }^{3}$ Ali Sameer Abdulghani Altaee, ${ }^{1}$ Katia Steiger, ${ }^{5}$ Marina Lesina, ${ }^{1}$ \\ Roland Rad, ${ }^{1,4}$ Maximilian Reichert, ${ }^{1}$ Guido von Figura, ${ }^{1}$ Jens T. Siveke, ${ }^{6,7,8}$ Roland M. Schmid, ${ }^{1,7}$ \\ and Clara Lubeseder-Martellato \\ ${ }^{1}$ Klinik und Poliklinik für Innere Medizin II, Technical University of Munich, Germany. ${ }^{2}$ Department of Histology and \\ Embryology, Manisa Celal Bayar University, Turkey. ${ }^{3}$ Department of Biology, McMaster University, Hamilton, Ontario, \\ Canada. ${ }^{4}$ Institute of Molecular Oncology and Functional Genomics and ${ }^{5}$ Institute of Pathology, Technical University of \\ Munich, Munich, Germany. ${ }^{6}$ Institute for Developmental Cancer Therapeutics, West German Cancer Center, University \\ Hospital Essen, Essen, Germany. 'Division of Solid Tumor Translational Oncology, German Cancer Consortium (DKTK) \\ partner site Essen, Essen, Germany. ${ }^{8}$ German Cancer Research Center (DKFZ), Heidelberg, Germany.
}

\begin{abstract}
Several studies have suggested an oncogenic role for the neural Wiskott-Aldrich syndrome protein (N-WASP, encoded by the Wasl gene), but thus far, little is known about its function in pancreatic ductal adenocarcinoma (PDAC). In this study, we performed in silico analysis of WASL expression in PDAC patients and found a correlation between low WASL expression and prolonged survival. To clarify the role of Wasl in pancreatic carcinogenesis, we used 2 oncogenic Kras-based PDAC mouse models with pancreas-specific Wasl deletion. In line with human data, both mouse models had an increased survival benefit due to either impaired tumor development in the presence of the tumor suppressor Trp53 or the delayed tumor progression and senescent phenotype upon genetic ablation of Trp53. Mechanistically, loss of Wasl resulted in cell-autonomous senescence through displacement of the N-WASP binding partners WASP-interacting protein (WIP) and p120ctn; vesicular accumulation of CSK3 $\beta$, as well as YAP1 and phosphorylated $\beta$-catenin, which are components of the destruction complex; and upregulation of $C d k n 1 a(p 21)$, a master regulator of senescence. Our findings, thus, indicate that Wasl functions in an oncogenic manner in PDAC by promoting the deregulation of the $\mathrm{p} 120$-catenin/ $\beta$-catenin/p21 pathway. Therefore, strategies to reduce $\mathrm{N}$-WASP activity might improve the survival outcomes of PDAC patients.
\end{abstract}

Authorship note: AHS, JD, ZD, and KS contributed equally to this work.

Conflict of interest: The authors have declared that no conflict of interest exists.

Copyright: (c) 2020, American Society for Clinical Investigation.

Submitted: January 9, 2019

Accepted: April 22, 2020

Published: May 21, 2020

Reference information: JCI Insight. 2020;5(10):e127275.

https://doi.org/10.1172/jci.

insight.127275.

\section{Introduction}

Activating mutations of the Kras oncogene are the driver mutations for pancreatic ductal adenocarcinomas (PDACs), which is one of, if not the, most lethal human cancer with a 5-year survival rate under $5 \%(1)$. In addition to Kras-activating mutations, several genetic alterations, such as inactivation of the tumor suppressor genes Trp53 and Cdkn2a(p16), also accumulate during the malignant transformation of the pancreas (2), which accelerates the progression of PDAC. Several studies suggest that PDAC develops through preneoplastic lesions originating from acinar-to-ductal metaplasia (ADM) (3-5). We previously showed that $\mathrm{ADM}$ depends on an increase in fluid-phase endocytosis (6) - a receptor-independent endocytic pathway characterized by fluid phase markers as cargos. We further showed that this ADM is dependent on the neural Wiskott-Aldrich syndrome protein (N-WASP, encoded by the Wasl gene). N-WASP is an indicator of poor prognosis in several cancers and has been implicated in the regulation of metastasis via the promotion of cell migration and remodeling of the extracellular matrix (6-11). At the cellular level, N-WASP interacts with components of the actin cytoskeleton, including the ARP2/3 complex and CDC42, as well as with PIP2 $(12,13)$. Notably, actin polymerization has been shown to be crucial for PDAC development, and both CDC42 and PIP2 are effectors of KRAS (14-16). N-WASP further interacts through its VCA and WH1 domains with p120-catenin (p120ctn) and the WASP-interacting protein (WIP), respectively (12, 13), both of which were recently implicated in PDAC aggressiveness and progression (17-19). Therefore, $\mathrm{N}$-WASP may function as a driver for PDAC development and progression. However, little is known about 
the role of N-WASP in PDACs. Taking advantage of the reproducible kinetics of tumor formation in mouse models of PDAC, we investigated the role of N-WASP expression in pancreatic cancer development and progression using genetic and molecular approaches.

Herein, we show that Wasl deletion in 2 mouse models of PDAC driven by oncogenic Kras leads to a survival benefit. Similarly, patients stratified for low WASL expression showed improved survival, underlying the relevance of the murine model to the human disease. Wasl deletion impaired tumor development in the presence of the tumor suppressor Trp53 and led to delayed tumor progression in the absence of Trp53. The biallelic loss of Wasl in the latter model also led to cell-autonomous senescence in pancreatic tumor cells characterized by Senescence-associated- $\beta$-galactosidase (SA- $\beta$-galactosidase) activity and $C d k n 1 a(p 21)$ upregulation. We also show a displacement of the N-WASP binding partners WIP and p120ctn upon deletion of Wasl, which resulted in increased endocytosis - linked to accumulation of the components of the $\beta$-catenin destruction complex YAP1 and dephosphorylated, inactive $\beta$-catenin in tumor cells. The induction of nuclear p120ctn combined with the inhibition of endocytosis rescued the senescent phenotype observed in Wasl-depleted cells. This suggests that Wasl functions, in part, in PDAC development by regulating p120ctn localization and by inhibiting endocytosis. Overall, this study demonstrates a tumor-promoting role for Wasl in PDAC development via regulation of protein stability and subcellular localization of components of the $\mathrm{p} 120 \mathrm{ctn} / \beta$-catenin signaling pathway.

\section{Results}

Wasl is involved in PDAC outcome. Wasl, encoding for N-WASP, is upregulated in both human and mouse PDAC models $(6,7)$ and has primarily been associated with enhanced tumor invasion. To determine the clinical relevance of Wasl in human PDAC, we stratified human PDAC samples for low and high WASL expression, and we observed a correlation between low WASL expression and increased survival in PDAC patients (Figure 1A). To better understand the function of Wasl in PDAC, we generated a pancreatic conditional Wasl-KO mice in the Ptfla ${ }^{\mathrm{Cre} /+} \mathrm{Kras}^{\mathrm{LSL}-\mathrm{Gl2D} /+}$ mouse model of PDAC (20) to generate Ptfla $^{\text {Cre/+ }}$ Kras $^{L S L-G 12 D /+}$ Was $^{\Delta \text { Panc }}$ mice (hereafter CK and CK-N ${ }^{\Delta \text { Panc }}$ mice, respectively). At 4 weeks of age, CK-N ${ }^{\triangle P a n c}$ pancreata showed large areas of edema, with infiltrating cells and fatty metaplasia, while CK mice pancreata exhibited large areas with acinar tissue and focal metaplastic lesions (Figure 1, B and D). Consistent with the gross phenotype, $\mathrm{CK}-\mathrm{N}^{\Delta \mathrm{Panc}}$ mice displayed an increase in the amount of pancreatic fat (Figure 1D) and an impressive infiltration of F4/80-positive macrophages, along with mast cells (Figure 1C and Supplemental Figure 1, A and B; supplemental material available online with this article; https:// doi.org/10.1172/jci.insight.127275DS1). These results were reminiscent of a pancreatitis-like phenotype. Notably, the infiltrating macrophages decreased with age, reaching a level below that present in the control terminal CK mice (Supplemental Figure 1B). In agreement with the inflammatory phenotype, CK-N $\mathrm{N}^{\Delta \mathrm{Pan}}$ expressed REG3A (Supplemental Figure 1C), which is upregulated during pancreatitis and is involved in $\mathrm{ADM}$ (21); CK-N $\mathrm{N}^{\Delta \text { Panc }}$ also expressed high active $\beta$-catenin levels, which is activated during pancreatitis (22) (Supplemental Figure 1, D and E). However, CK-N ${ }^{\Delta \text { Panc }}$ mice do not develop ADM at 3 months of age (6), which led us to conclude that lack of Wasl blocks ADM downstream of REG3A and $\beta$-catenin. To unravel the molecular effect of Wasl depletion, we performed RNA sequencing (RNAseq) of the pancreata of 4-week-old CK and CK-N ${ }^{\Delta \text { Panc }}$ mice. The differentially expressed genes with an adjusted $P$ value of < 0.02 (Supplemental Table 1) were subjected to gene set enrichment analysis using the program Enrichr (23, 24). In agreement with our histological data, $C K-N^{\Delta P a n c}$ pancreatic tissues displayed an enrichment of genes involved in pancreatitis and the immune system (Figure 1E and Supplemental Table 2) and a downregulation of genes involved in the DNA replication pathway (Figure 1E and Supplemental Table 2). Notably, pancreatitis induces injury of acinar cells that has been described to lead to fatty degeneration (25), which may explain the observed fatty metaplasia. Gene Set Enrichment Analysis (GSEA; https://www.gseamsigdb.org/gsea/index.jsp) also revealed an upregulation of adipogenesis and mast cell immunity, which were consistent with the observed "fatty" phenotype seen in the CK-N ${ }^{\Delta \mathrm{Panc}}$ mice, as well as an upregulation of inflammatory responses and IL-6 signaling (Figure 1F). In line with the increased amount of active $\beta$-catenin expression observed in CK-N ${ }^{\Delta P a n c}$ pancreatic tissues, our RNAseq data reveal increased expression of several $\beta$-catenin gene targets (Supplemental Table 3), which were validated by reverse transcription PCR (RT-PCR) (Supplemental Figure 1G). Notably, we observed downregulation of the $\beta$-catenin target $M y c$ in CK-N ${ }^{\Delta \text { Panc }}$ pancreatic tissues, along with downregulation of MYC targets, as shown by GSEA (Supplemental Figure $1 \mathrm{~F}$ and Supplemental Table 3). Since inflammation and IL-6 signaling have been linked 
A

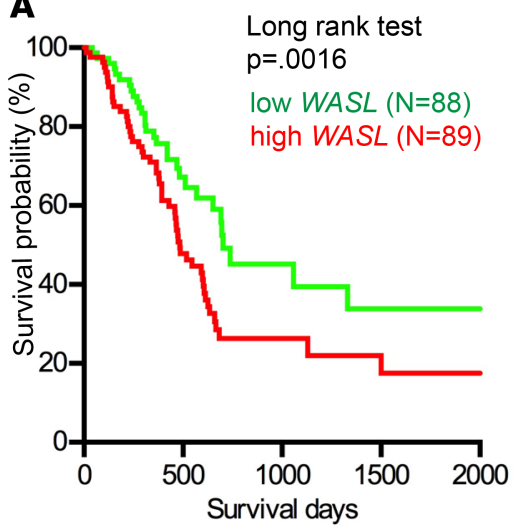

E



B

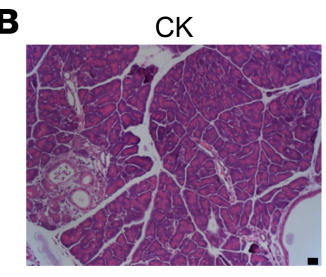

C

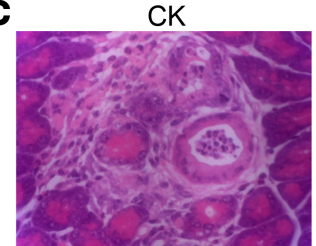

$\mathrm{CK}^{-\mathrm{N}^{\Delta \mathrm{Panc}}}$

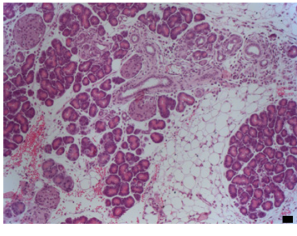

$\mathrm{CK}^{\mathrm{N}}{ }^{\Delta \mathrm{Panc}}$

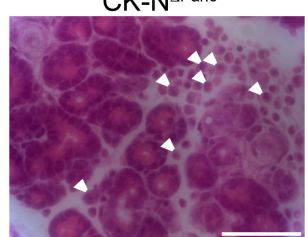

D

D $\square$ CK $\square$ CK-NAPanc

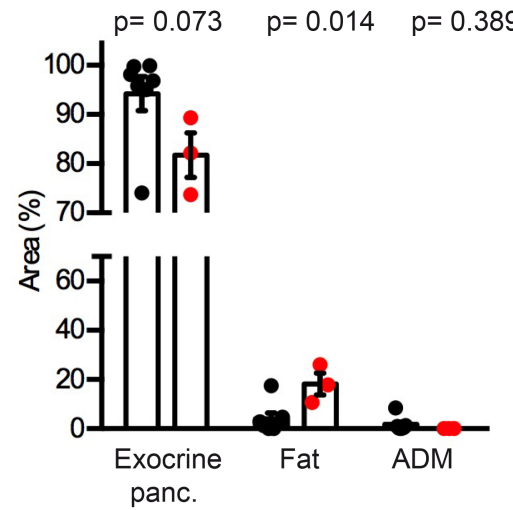

$\mathbf{F}$

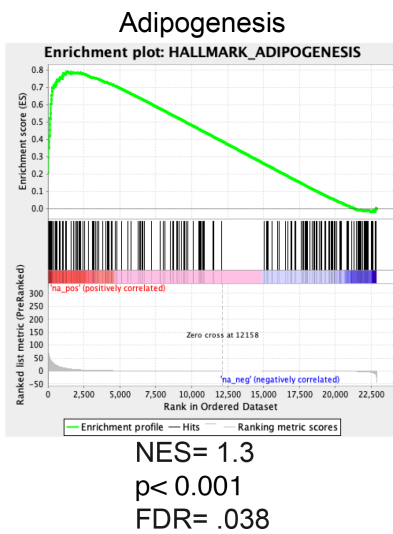

G Long rank test

CK-N ${ }^{\Delta \text { Panc }} / C K-N^{\text {het }} p=.0006$

CK-N ${ }^{\Delta P a n c} / C K \quad p<.0001$

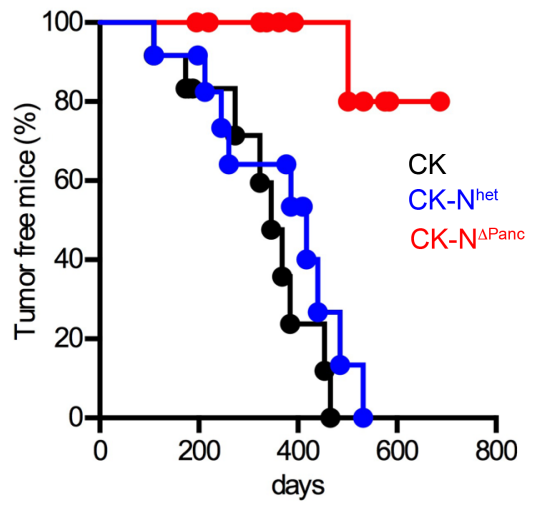

Mast cell immunity Enrichment plot:
GO_MAST_CELL_MEDIATED_IMMUNITY

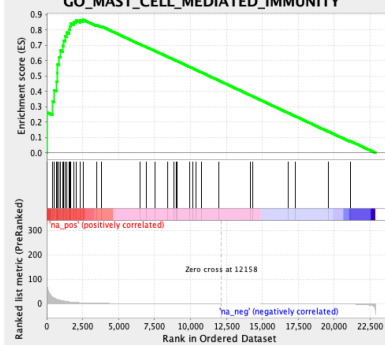

$\mathrm{NES}=1.4$

$\mathrm{p}=.007$

$\mathrm{FDR}=.022$

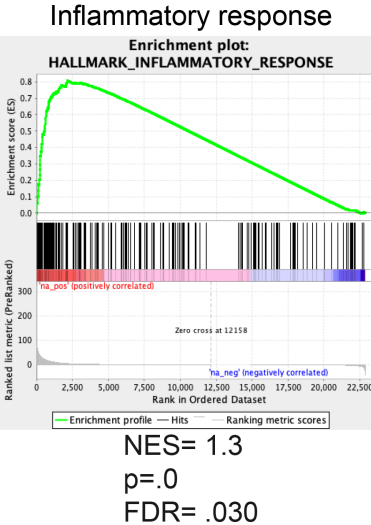

Inflammatory response

Enrichment plot:
HALLMARK_INFLAMMATORY_RESPONS

H

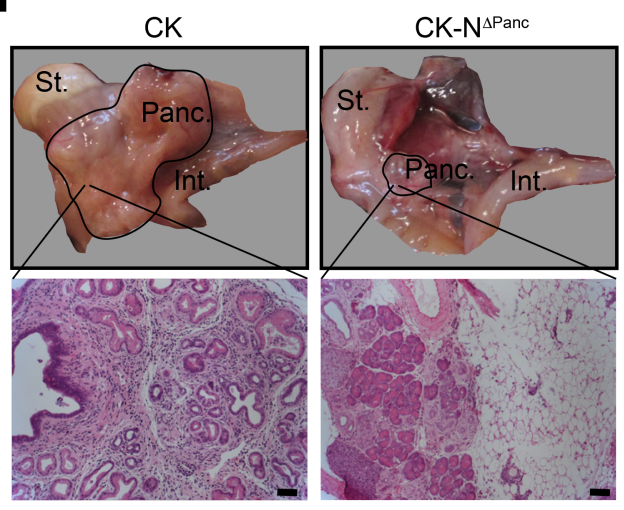

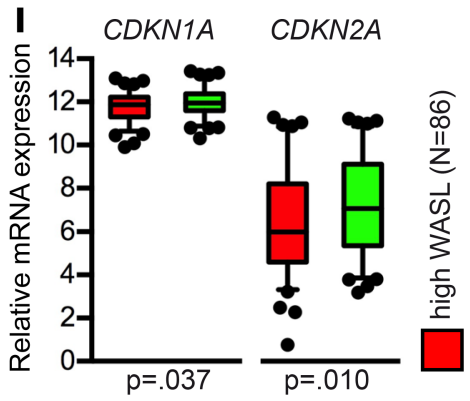

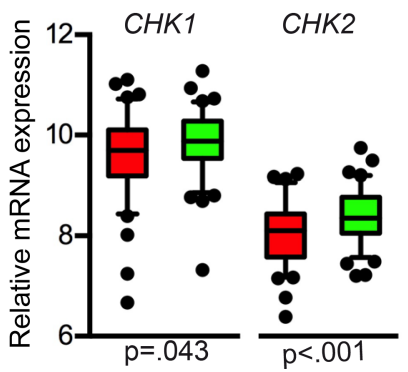

IL6 signaling Enrichment plot:
HALLMARK_IL_JAK_STAT3_SIGNALING

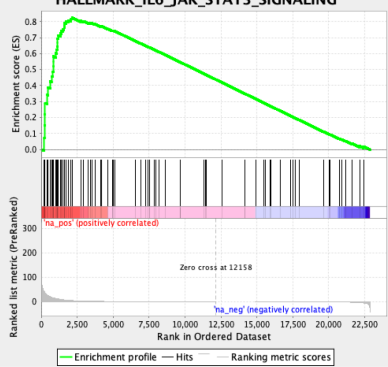

$\mathrm{NES}=1.3$

$\mathrm{P}=003$

$\mathrm{FDR}=.028$ 
Figure 1. Low WASL correlates with improved survival, and loss of Wasl in oncogenic Kras-driven mouse model for PDAC impairs tumor development. (A) Kaplan-Meier survival curve of PDAC patients stratified for WASL expression using the UCSC Xena genomic browser. (B) Representative H\&E staining of pancreata of 4-week-old mice. (C) H\&E stainings; arrowheads highlight mast cells. Scale bars: $50 \mu \mathrm{M}$. (D) Morphometric quantification of the amount of ADM lesions, exocrine pancreas, and fat within the pancreatic tissue in 4-week-old animals. CK $(n=7)$ and CK-N ${ }^{\Delta \text { Panc }}(n=3)$. Data represent mean \pm SEM. Student's $t$ test. (E) RNAseq was performed from 4 mice per group. The bar graph from the top upregulated and downregulated pathways (combined score: $P$ value multiplied by $z$ score) in 4-week-old CKN ${ }^{\Delta \text { Panc }}$ mice. The pathways were obtained after running the differentially regulated genes ( $\left.n=155\right)$ listed in Supplemental Table 1 through the gene set enrichment program Enrichr. (F) Enrichment plots generated by the GSEA tool showing the signatures that were enriched in the CK-N ${ }^{\Delta P a n c}$ pancreata. (C) Kaplan-Meier tumor-free curves for CK $(n=12)$, CK- $\mathrm{N}^{\text {het }}(n=12)$, and CK-N $\mathrm{N}^{\Delta \text { Panc }}(n=12)$ mice. (H) Upper panels show macroscopic pictures of organs of endpoint mice. Note the tiny pancreas in the CKP-N ${ }^{\Delta P a n c}$ mouse. St., stomach; Panc., pancreas; Int., intestine. The lower panels show representative H\&E stainings. Scale bars: $50 \mu \mathrm{M}$. (I) PDAC patients were stratified for WASL expression using the UCSC Xena genomic browser, and expression levels of the indicated genes were compared. In the box plots, the whiskers represent the 5 th and 95th percentile, and the central line is the median of the data, with the remaining dots being outliers. Unpaired $t$ test with Welch's correction.

to oncogene-induced senescence (OIS) $(26,27)$ and $M y c$ is an essential downstream effector of oncogenic Kras in the pancreas $(28,29)$, and since pancreatitis is a factor promoting PDAC $(30,31)$, we monitored a cohort of CK-N ${ }^{\triangle P a n c}$ mice until endpoint.

CK control mice reproducibly develop PDAC at the age of about 1 year (20), and mice with deletion of 1 Wasl allele in this background (hereafter CK- ${ }^{\text {het }}$ ) developed tumors at a rate comparable with that of $\mathrm{CK}$ mice and with similar survival (Figure $1 \mathrm{G}$ and Supplemental Figure1H). Surprisingly, deletion of both Wasl alleles impaired tumor development (Figure 1H) and slightly improved survival (Supplemental Figure 1H) compared with CK mice. CK-N ${ }^{\triangle P a n c}$ mice developed pancreata with small areas of normal exocrine pancreas and adipose tissue surrounding sparse islets of Langerhans, suggesting lipomatosis of the exocrine tissue (Supplemental Figure 1, I and J). The remaining exocrine pancreas also had sparse cells showing SA- $\beta$-galactosidase activity (Supplemental Figure1K), which is the most accepted biomarker of senescence in vivo $(32,33)$. Consistent with these findings, low WASL expression in human patients correlates with increased expression of the senescence markers $C D K N 1 A$ and $C D K N 2 A$ and the checkpoint kinases $C H K 1$ and $C H K 2$ (Figure 1I). Thus, loss of Wasl impaired oncogenic Kras-driven tumor development by inducing an inflammatory and senescence transcriptional program coupled to a fatty metaplasia.

Concomitant Wasl and Trp53 loss improves survival of Kras-driven tumors and leads to tumors with senescence features. Since the CK- $\mathrm{N}^{\Delta \mathrm{Panc}}$ mice did not develop tumors, we next analyzed a mouse model with a more aggressive pancreatic tumor. As a model for fast-developing PDAC, we used the Ptfla ${ }^{\mathrm{Cre} /+} \mathrm{Kras}^{L S L-G 12 \mathrm{D} /+}$ $\operatorname{Trp} 53^{\text {月/fl}}$ mouse model (34) (hereafter CKP), which has a pancreas-specific homozygous deletion of Trp53 that results in loss of $\mathrm{p} 53$ protein expression (Supplemental Figure 2A) and which develops PDAC within 4-6 weeks in a setting of homozygous Trp53 deletion. We then assessed the impact of heterozygous and homozygous deletion of Wasl in the pancreas of the CKP mouse model. CKP mice lacking 1 Wasl allele (hereafter CKP-N $\mathrm{N}^{\text {het }}$ ), developed ductal adenocarcinomas, defined by the presence of neoplastic glandular cells in a dense fibrous stroma, and were indistinguishable from CKP mice (Supplemental Figure 2B). Additionally, CKP and CKP-N ${ }^{\text {het }}$ mice had a comparable life span (Supplemental Figure 2C). Thus, we used the CKP-N $\mathrm{N}^{\text {het }}$ mice as a control cohort. Homozygous deletion of Wasl in CKP mice (hereafter CKP$\mathrm{N}^{\Delta \mathrm{Panc}}$ ) was validated by immunohistochemical staining for N-WASP (Figure 2A), as well as by RT-PCR analysis of Wasl expression (Supplemental Figure 2D). The CKP-N ${ }^{\Delta P a n c}$ mice had a significantly improved survival compared with CKP-N ${ }^{\text {het }}$ mice (Figure 2B), consistent with the improved survival observed in human PDAC patients with low Wasl-expressing pancreatic tumors. Similar to the CK-N ${ }^{\Delta P a n c}$ mice, the pancreatic tissues of the CKP-N ${ }^{\Delta P a n c}$ mice also exhibited high levels of lipomatosis (Figure 2D and Supplemental Figure 2, E and F). We also observed that the CKP-N $\mathrm{N}^{\Delta P a n c}$ tumors were predominantly ductal adenocarcinomas with frequent mesenchymal features (50\% of CKP-N ${ }^{\Delta P a n c}$ mice, compared with $20 \%$ of CKP-N ${ }^{\text {het }}$; Supplemental Table 4), characterized by TWIST- and ZEB1-expressing fibroblasts and macrophages (Supplemental Figure 2, G-I). However, the tumors formed by the CKP-N ${ }^{\Delta P a n c}$ mice had a lower proliferation index, as shown by the decreased Ki67 staining (Figure 2C). This suggests that the improved survival seen in the CKP-N ${ }^{\Delta P a n c}$ mice is due to delayed tumor development.

Next, we sought to determine the mechanism via which loss of Wasl expression in the CKP mice background contributed to improved survival. Oncogenic Kras induces cellular senescence, which is suppressed by loss of $\operatorname{Trp} 53(27,33,35)$. Accordingly, the lesions in tumors from CK mice were positive for the senescence marker SA- $\beta$-galactosidase, and those in CKP tumors were negative (Figure 2E). Notably, deletion of both Wasl alleles in the CKP mice background led to the appearance of SA- $\beta$-galactosidase-positive 
A

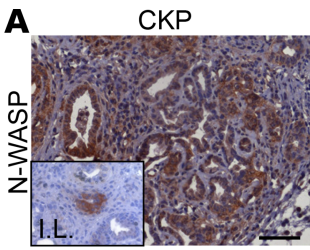

B


$\mathbf{E}$

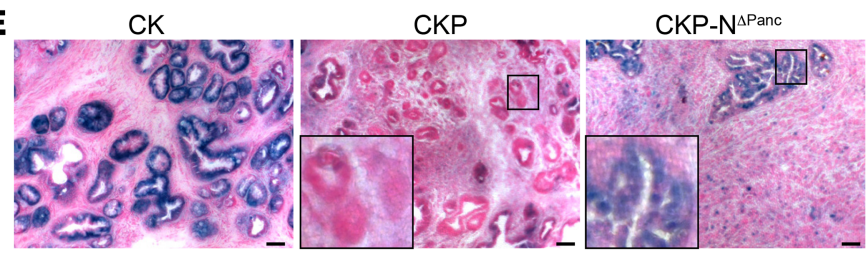

Senescence associated $\beta$-galactosidase activity
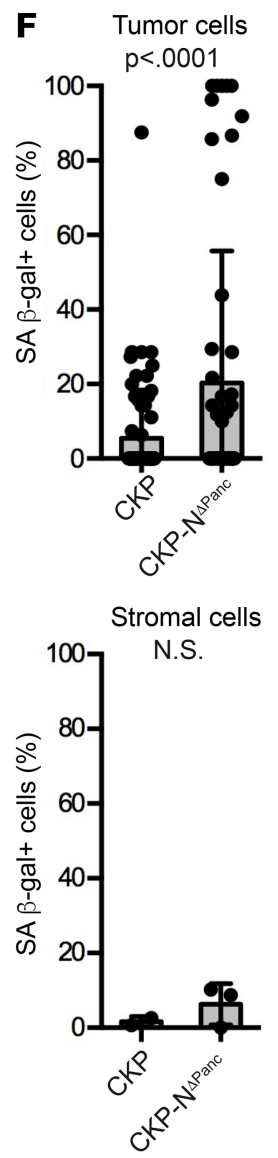

Figure 2. CKP-N ${ }^{\Delta \text { Panc }}$ mice have improved survival and develop tumors that express senescent markers. (A) Immunohistochemical staining showing the lack of N-WASP expression in pancreata of CKP-N ${ }^{\Delta \text { Panc }}$ mice. Scale bars: $50 \mu \mathrm{M}$. The inserts show the islets of Langerhans (I.L.) as an internal positive control for N-WASP staining. (B) Kaplan-Meier survival curves for mice of the indicated genotypes. CKP-N ${ }^{\text {het }}(n=12)$ and CKP-N $\mathrm{N}^{\Delta \text { Panc }}$ $(n=10)$. Log-rank test. (C) Murine tumor specimen were stained for the proliferation marker Ki67. The number of Ki67-positive tumor cells was quantified per relative area. Data represent mean \pm SEM; Student's $t$ test. (D) Representative pictures of tumors developed by the indicated mice. Scale bars: $50 \mu \mathrm{M}$. (E) SA- $\beta$-galactosidase staining of cryosections of murine tumors. Scale bars: $50 \mu \mathrm{M}$. Inserts show magnification of the black boxes. (F) The amount of SA- $\beta$-galactosidase-positive cells was quantified in the tumor cell and stromal cell compartment. Data represent the mean of 2 mice per group \pm SEM. Student's $t$ test.

(G) The expression of the indicated genes was analyzed by RT-PCR. Data represent the mean of 4 of 5 tumors per group \pm SEM. Student's $t$ test. (H) Enrichment plots generated by the GSEA tool. The IL-6/JAK/STAT3 and TNFA signaling via NF- $\kappa B$ signaling pathways, as well as the IFNG response signatures, were enriched in the CKP-N $\mathrm{N}^{\triangle \mathrm{Panc}}$ tumors.

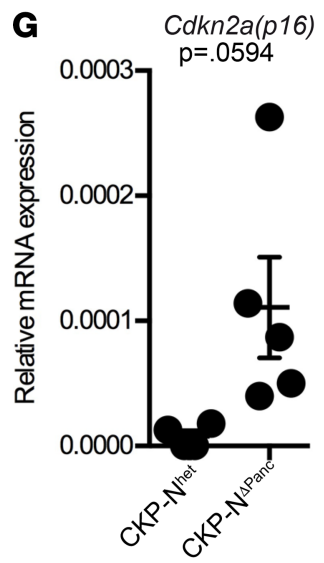

H

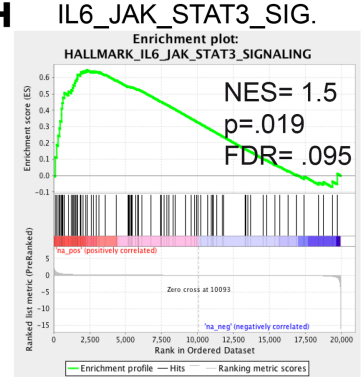

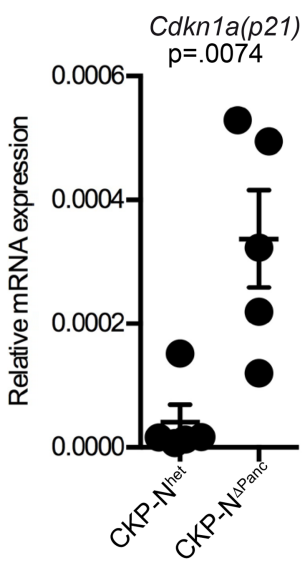

INTERFERON_GAMMA_R. Enrichment plot:
HALLMARK INTERFERON_GAMMA_RESPONSE

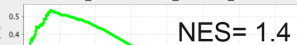
$\mathrm{p}=.027$ $F D R=.175$

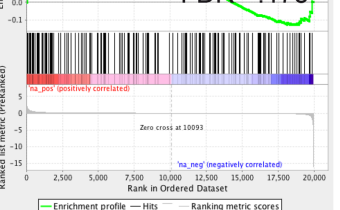

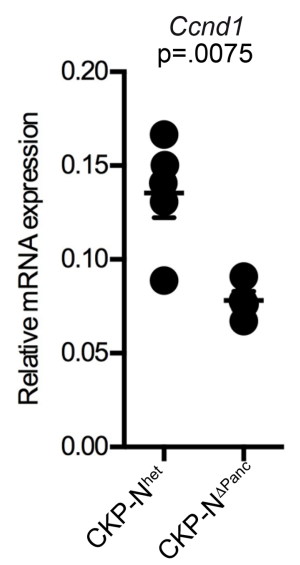

TNFA_SIG._VIA_NFKB HALLMARK_TNRA__SICNALINC_VIA_NFKB NES $=1.4$ $\mathrm{p}=.017$ $\mathrm{FDR}=.20$




senescent tumor cells (Figures 2, E and F). The CKP-N ${ }^{\Delta \text { Panc }}$ tumors also exhibited increased Cdkn1a(p21) and Cdkn2a expression, along with low Ccnd1 levels (Figure 2G) and high p16 protein expression (Supplemental Figure 2, J and K). Remarkably, these tumors exhibited a molecular phenotype similar to the tumors of human PDAC patients with low WASL expression (compared with Figure $1 \mathrm{H}$ ). Together, these data indicate that lack of Wasl expression overcomes the escape of senescence phenotype observed in the tumors of CKP mice models lacking Trp53.

CKP-N $^{\Delta P a n c}(n=7)$ and CKP-N ${ }^{\text {het }}(n=6)$ tumors were next subjected to RNAseq analysis, which identified several differentially expressed genes in the CKP-N ${ }^{\Delta P a n c}$ tumors, including Wips $2 / C c n 5$ (Supplemental Table 5) that were validated by RT-PCR analysis (Supplemental Figure 2L). GSEA revealed that the CKP$\mathrm{N}^{\Delta \text { Panc }}$ tumors were enriched in genes involved in IL- 6 and TNF- $\alpha$ signaling, as well as increased IFN- $\gamma$ response compared with controls (Figure $2 \mathrm{H}$ ). Notably these genes are all linked to pathways implicated in a senescence-associated secretory phenotype (SASP) (36).

To investigate if the senescence phenotype observed in $C K P-N^{\Delta \text { Panc }}$ tumors is cell autonomous, we used 2 approaches. First, we established cell lines from tumors with different Wasl allelic status (CKP, CKP$\mathrm{N}^{\text {het }}$, and $\left.\mathrm{CKP}-\mathrm{N}^{\Delta \mathrm{Panc}}\right)$. Characterization of these cell lines showed a strong SA- $\beta$-galactosidase activity in CKP-N ${ }^{\Delta \text { Panc }}$ cells only (Figures 3, A and B). Similar to the primary tumors, Cdkn1a(p21) expression was increased concomitant with a slightly decreased $C$ cnd1 expression (Figure 3C) and low proliferation rates (Supplemental Figure 3A) in CKP-N ${ }^{\Delta P a n c}$ cells compared with CKP and CKP-N ${ }^{\text {het }}$ cells. Analysis of the culture supernatants using a cytokine profiler revealed increased secretion of some components of the SASP (36), including GM-CSF, KC (CXCL1), and IL-1ra (Figure 3, D and E) in the CKP-N ${ }^{\Delta \text { Panc }}$ cells. This is consistent with the lower levels of the SASP-associated genes GM-CSF (CSF2), CXCL1, and CCL5 $(M C P-2)$ (Supplemental Figure 3B) observed in PDAC patients with low WASL expression. These data, thus, support a senescent and slow proliferating phenotype of the CKP-N $\mathrm{N}^{\Delta \text { Panc }}$ cells due to lack of $W A S L$ expression. In the second approach, we isolated tumor organoids (37-39) from both CKP and CKP-N ${ }^{\triangle P a n c}$ mouse models. The CKP-N ${ }^{\Delta P a n c}$ organoids exhibited delayed growth in 3D-Matrigel cultures (Figure $3 \mathrm{~F}$ ), along with sparse but thicker organoids walls (Figure 3G and Supplemental Figure 3C) and impaired size gain through passaging compared with CKP organoids (Supplemental Figure 3D), indicating the presence of senescent features in the CKP-N ${ }^{\triangle P a n c}$ organoids. Altogether, these data demonstrate that lack of Wasl delays tumor cell growth and induces senescence in pancreatic tumor cells in a cell-autonomous manner and bypasses the effect of Trp53 loss.

Loss of Wasl leads to delocalization of N-WASP protein binding partners. Nuclear N-WASP has recently been linked to regulation of senescence (10). However, no nuclear N-WASP staining was observed in CKP and CKP-N ${ }^{\text {het }}$ tumors (Figure 2A). Therefore, to understand the mechanism driving the senescence effect of N-WASP loss, we examined its binding partners. N-WASP interacts with WIP and p120ctn through its WH1 and VCA domains, respectively $(40,41)$. WIP is expressed in all CKP-N ${ }^{\text {het }}$ and CKP-N ${ }^{\Delta P a n c}$ cancers with comparable protein levels (Supplemental Figure 4A). However, WIP hardly showed a membranous staining pattern in CKP-N ${ }^{\triangle P a n c}$ mouse tumors compared with its CKP and CKP-N ${ }^{\text {het }}$ counterparts (Figure 4A, Table 1, and Supplemental Figure 4B). In glioblastomas (GB), WIP is involved in the control of the endocytic/endosomal system and in the sequestration of GSK3, which is a component of the $\beta$-catenin destruction complex (17). Therefore, we next analyzed the endosomal function of CKP-N ${ }^{\triangle P a n c}$ cells and detected increased levels of horseradish peroxidase (HRP) uptake, indicative of increased endocytosis (Figure 4B), along with a GSK3 $\beta$ vesicular staining (Figure $4 \mathrm{C}$ ). Furthermore, GSK3 $\beta$ staining was weak in CKP-N ${ }^{\Delta P a n c}$ tumor tissues compared with CKP-N ${ }^{\text {het }}$ control tumor tissues (Supplemental Figure 4C). Based on these findings, we hypothesized that there is endosomal sequestration of destruction complex components in PDAC, akin to GB. We next analyzed the expression of further components of the destruction complex including of phosphorylated $\beta$-catenin and YAP1 (42). Phosphorylated $\beta$-catenin and YAP1 were enriched in CKP-N ${ }^{\Delta \text { Panc }}$ tumors (Figure 4, D, F, and G), while the levels of active (nonphosphorylated) $\beta$-catenin and MYC (a $\beta$-catenin target gene) were decreased, and there was a downregulation of MYC targets (Figure 4E and Supplemental Figure 4D). We again stratified PDAC patients with high and low WASL-expressing tumors using the Xena browser and observed that low WASL expression correlated with low expression of the destruction complex components (Figure $4 \mathrm{H})$, consistent with our findings in CKP-N $\mathrm{N}^{\triangle \mathrm{Panc}}$ murine tumors. Thus, our data support a mechanism of endosomal sequestration of the destruction complex components in CKP-N $\mathrm{N}^{\Delta \mathrm{Panc}}$ tumors.

In addition to WIP delocalization, we observed a decrease in the nuclear fraction of p120ctn in CKP$\mathrm{N}^{\Delta \text { Panc }}$ tumor cells both by immunofluorescence analysis and Western blot analysis (Figures 5, A-C). 
A

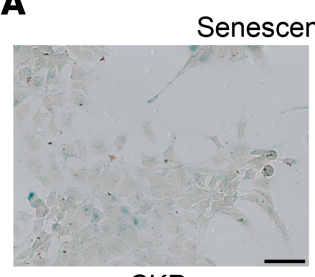

CKP

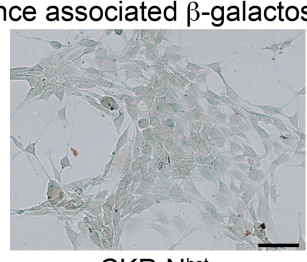

CKP-N $^{\text {het }}$

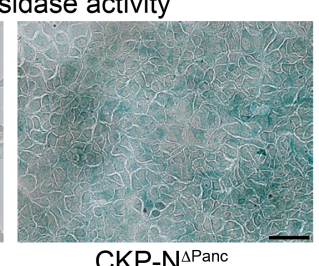

$\mathrm{CKP}^{-\mathrm{N}^{\Delta \mathrm{Panc}}}$
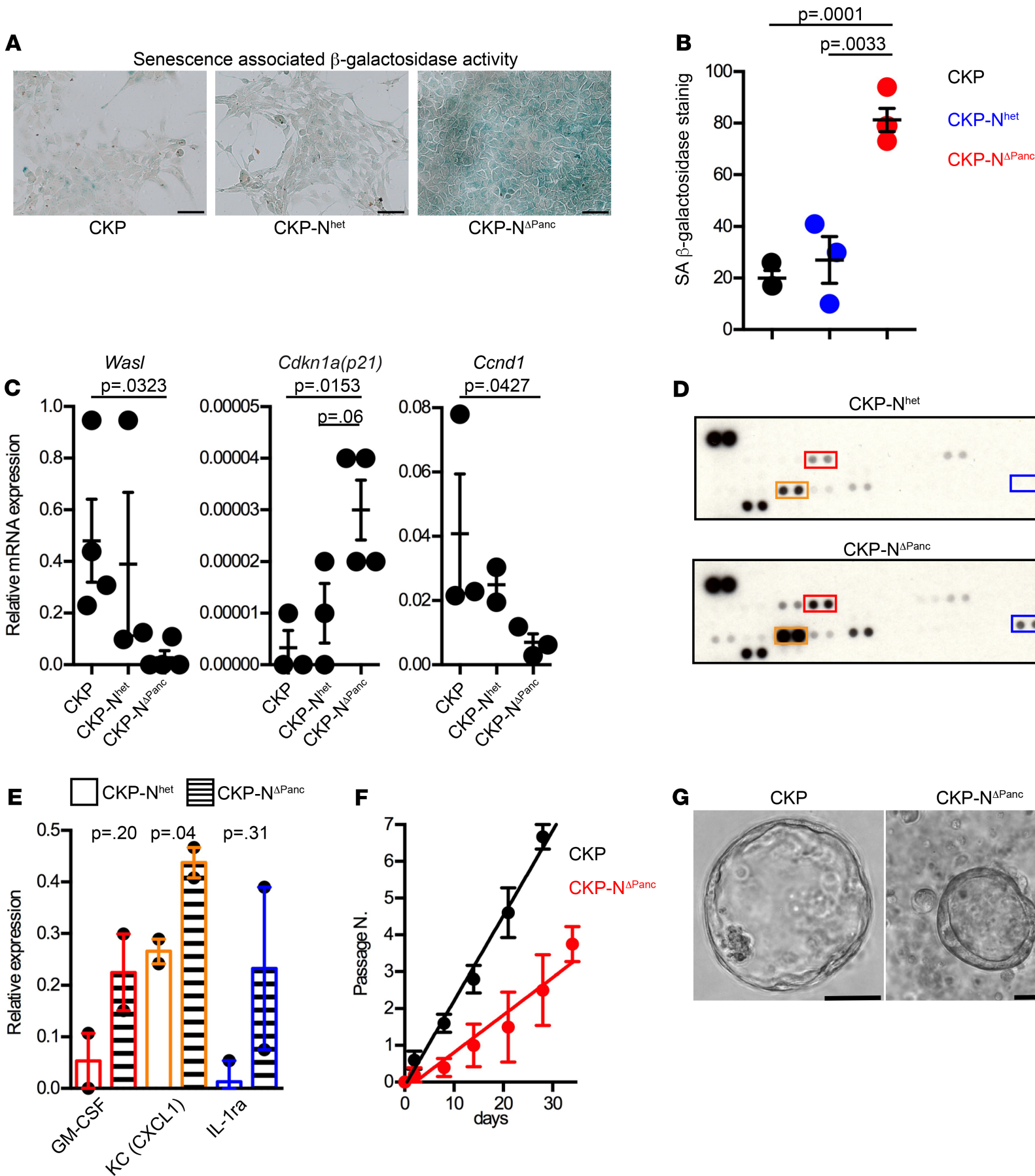

$\mathbf{F}$



D
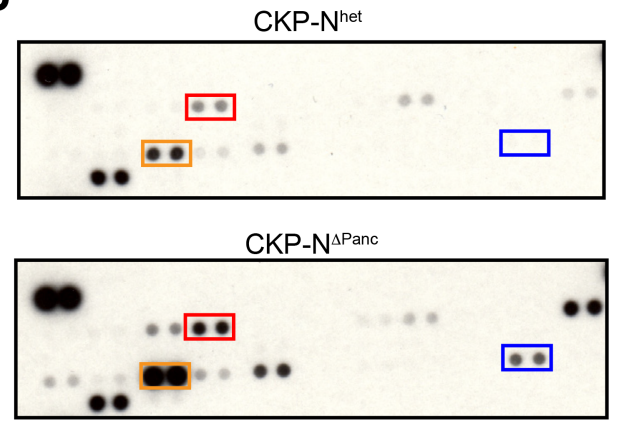

G

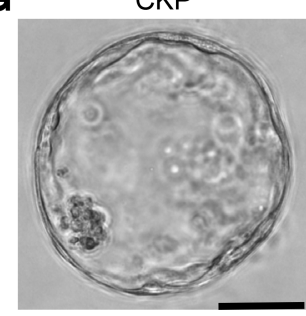

CKP-N ${ }^{\Delta P a n c}$

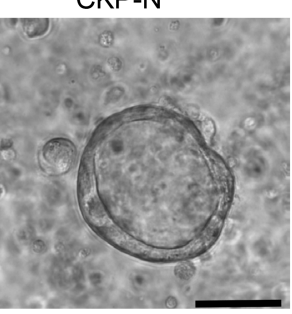

Figure 3. Primary tumor cells isolated from CKP-N $\mathbf{N}^{\Delta \text { Panc }}$ mice have a senescent phenotype. $(\mathbf{A}$ and $\mathbf{B})$ Cell lines from CKP $(n=3)$, CKP-N $\mathrm{N}^{\text {het }}(n=3)$, and CKP-N ${ }^{\Delta P a n c}(n=4)$ were grown on a thin layer of collagen and stained for SA- $\beta$-galactosidase, quantified in B. Data represent mean \pm SEM; Kruskal-Wallis 1-way ANOVA test $(P=0.01)$ followed by Dunn's multiple comparisons test. (C) Analysis of gene expression by RT-PCR of primary tumor cell lines isolated from mice of the indicated genotypes ( 3 of 4 cell lines per group). Data represent mean \pm SEM; Kruskal-Wallis 1-way ANOVA test (Wasl, $P=0.021 ; C d k n 1 a, P=0.029, C c d n 1, P=0.06$ ) followed by Dunn's multiple comparisons test. (D) Conditioned media were collected from CKP-N ${ }^{\text {het }}$ and CKP-N $\mathrm{N}^{\Delta \mathrm{Panc}}$ cells, which were grown on a thin layer of collagen for cytokine array analysis. The boxed regions on the representative blot indicate the differentially secreted cytokines. (E) Quantification of the boxed regions as described in $\mathbf{D}$. Data represent the mean of 2 cell lines per group \pm SEM. Student's $t$ test. (F and $\mathbf{G})$ Organoids generated from CKP $(n=5)$ and CKP-N $\mathrm{N}^{\triangle P a n c}(n=5)$ pancreata were isolated. The passage numbers in function of the time are shown. Data represent mean \pm SEM. (F) Organoids generated from CKP $(n=5)$ and CKP-N ${ }^{\Delta P a n c}(n=5)$ pancreata were cultivated. The passage numbers in function of the time are shown. Data represent mean \pm SEM. The slopes are substantially different indicating a slower growth of the CKP-N $\mathrm{N}^{\Delta \text { Panc }}$ organoids. (C) Representative bright-field images of organoids 14 days after isolation. The CKP-N $\mathrm{N}^{\Delta \mathrm{Panc}}$ organoids were not passaged during this period, while the CKP organoids were passaged 3 times. 
A

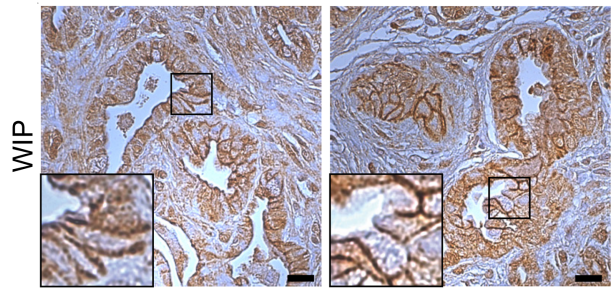

CKP-N ${ }^{\Delta P a n c}$



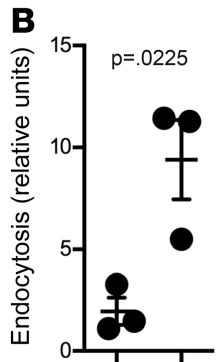
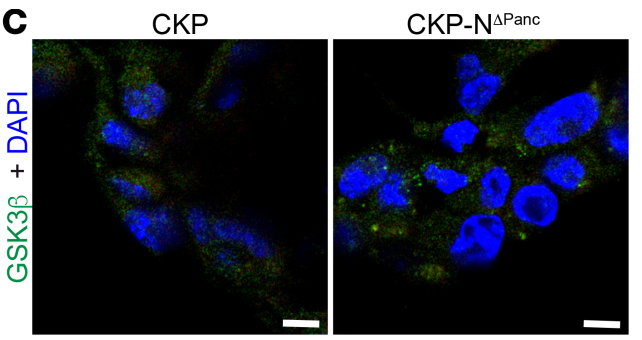

D



$\mathbf{F}$

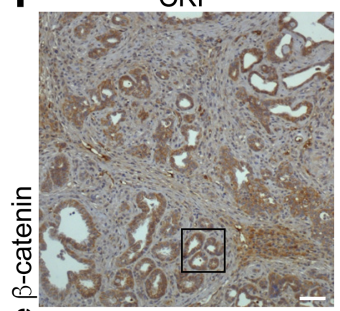

选

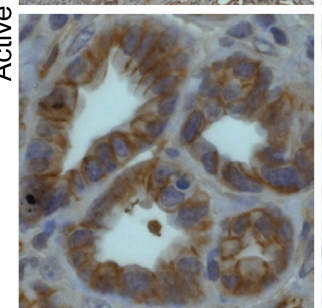

CKP-Nhet

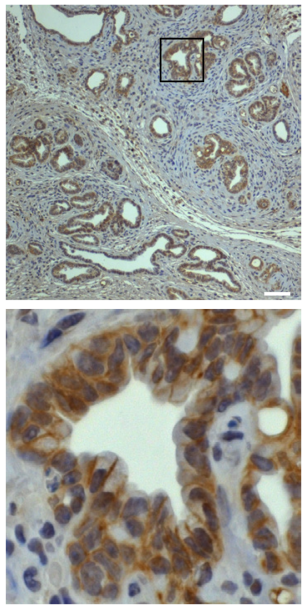

CKP-N ${ }^{\Delta P a n c}$

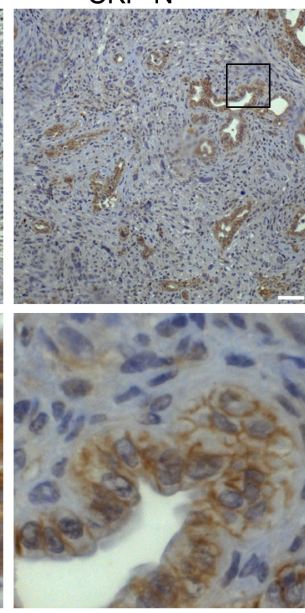

E
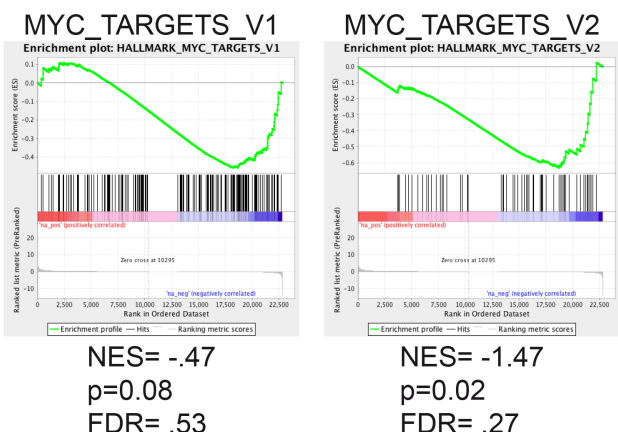

$\mathrm{FDR}=.27$
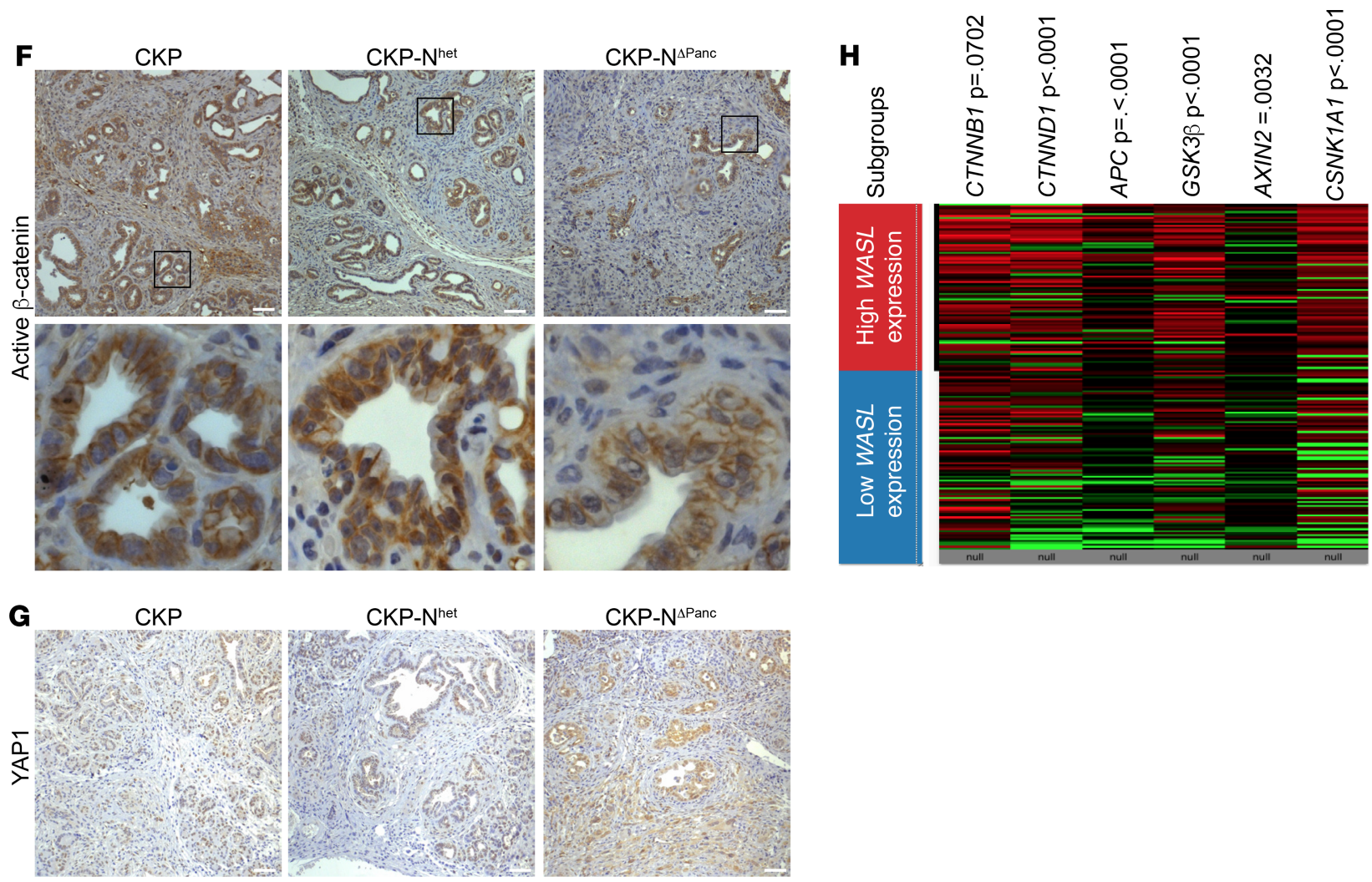

Figure 4. Lack of Wasl leads to the accumulation of components of the destruction complex and increased expression of inactive $\beta$-catenin. (A) Immunohistochemical staining for WIP in murine tumors. Scale bars: 50 M. Inserts show magnification of the black boxes. (B) Three cell lines per genotype were grown overnight; then, an HRP-endocytic assay was performed. The relative endocytosis level is shown. Each point represents the mean from 3 experiments, and the mean of 3 cell lines \pm SEM is shown. Student's $t$ test. (C) Cells were grown on a thin layer of collagen, and an immunofluorescence staining for GSK3 $\beta$ was performed. Nuclei are counterstained with DAPI. Scale bars: $10 \mu$ M. (D) Western blot analysis showing $\beta$-catenin phosphorylation at Ser33/37 and Thr41, as well as total YAP1. Actin and ERK1 + ERK2 were used as loading controls. (E) Enrichment plots 
generated by the GSEA tool. The signatures for MYC targets were enriched in the CKP-N ${ }^{\triangle P a n c}$ tumors. (F and G) Immunohistochemical stainings for active $\beta$-catenin and YAP1. Scale bars: $50 \mathrm{M}$. The right panels show magnification of the black boxes. (H) PDAC patients were stratified for WASL expression using the UCSC Xena genomic browser, and the expression levels of the indicated genes were compared. WASL high ( $n=86$ ), WASL low ( $n$ = 89). Unpaired $t$ test with Welch's correction.

Nuclear p120ctn has been shown to interact with, and regulates the transcriptional activity of, the multifaceted transcription factor KAISO (encoded by the Zbtb33 gene) that negatively regulates Wnt/ $\beta$-catenin signaling (43-46). Therefore, we examined KAISO expression in CKP-N ${ }^{\triangle P a n c}$ tumor cells. Notably, we detected increased KAISO protein expression in CKP-N ${ }^{\Delta \text { Panc }}$ tumors (Figure 5, D and E), as well as in the matched isolated cells, compared with controls (Supplemental Figure 5, A and B). However, there was no apparent increase in Kaiso gene expression (Supplemental Figure 5C), suggesting that Kaiso is posttranslationally regulated and/or stabilized in CKP-N ${ }^{\Delta P a n c}$ tumors. In addition, we detected less colocalization of KAISO with the active transcription marker $\mathrm{H} 3 \mathrm{~K} 4$ in $\mathrm{CKP}-\mathrm{N}^{\triangle \mathrm{Panc}}$ tumor cells compared with controls (Supplemental Figure 5, D and E), suggesting a displacement of KAISO from active promoter regions (47-49). We next assessed the endogenous p120ctn/KAISO interaction using immunoprecipitation assays and detected the p120ctn/KAISO complex in CKP-N ${ }^{\triangle P a n c}$ tumor cells (Supplemental Figure 5F).

In summary, lack of N-WASP in CKP tumors correlates with an enrichment of endocytosis concomitant with an accumulation of components of the $\beta$-catenin destruction complex and the transcription factor KAISO but with nuclear displacement of Kaiso's binding partner p120ctn.

Inhibition of endocytosis and increased nuclear p120ctn are required to rescue the phenotype of $C K P-N{ }^{\triangle P a n c}$ tumor cells. To confirm the involvement of the destruction complex and p120ctn in the phenotype of the CKP$\mathrm{N}^{\Delta \text { Panc }}$ tumors, we performed the following functional experiments. First, we treated CKP-N $\mathrm{N}^{\Delta \text { Panc }}$ tumor cells with endocytosis inhibitors. We included Cytochalasin D (which inhibits actin polymerization), the $\mathrm{Na}^{+} /$ $\mathrm{H}^{+}$exchanger ethyl-isopropyl-amiloride (EIPA), and the ionophore salinomycin. All treatments strongly induced cytoplasmic GSK3 $\beta$ localization concomitant with decreased total YAP1 protein expression (Figure 6, A and B). This result indicates the successful inhibition of the GSK3 $\beta$ endosomal sequestration. The 24-hour EIPA treatment downregulated $C$ cnd1 expression in the control cell lines, consistent with published studies (50) (data not shown). All treatments slightly upregulated Cdkn1a(p21) (Figure 6C) and downregulated Cond1 (Supplemental Figure 6A) but had no significant effect on the number of senescent cells (Figure 6D). Therefore, this treatment - although inducing a decrease of YAP1 in the CKP-N ${ }^{\triangle P a n c}$ tumor cells - was not able to rescue their senescence phenotype. Next, based on the observation of decreased nuclear p120ctn in CKP-N $\mathrm{N}^{\Delta \text { Panc }}$ tumors cells, we treated CKP-N $\mathrm{N}^{\triangle P a n c}$ tumor cells with leptomycin B (LMB) since p120ctn nucleocytoplasmic shuttling is sensitive to LMB treatment (Figure 6E) $(43,51)$. However, LMB treatment alone was not sufficient to rescue the senescence phenotype of CKP-N ${ }^{\Delta \text { Panc }}$ tumor cells (Supplemental Figure 6). We next treated CKP-N ${ }^{\Delta \text { Panc }}$ tumor cells with a combination of salinomycin and $\mathrm{LMB}$, which target both deregulated pathways in the CKP-N ${ }^{\Delta \text { Panc }}$ tumor cells. This sequential treatment of CKP-N $\mathrm{N}^{\Delta \text { Panc }}$ tumor cells rescued the senescent phenotype of CKP-N $\mathrm{N}^{\Delta \text { Panc }}$ tumor cells, as shown by the significant decrease of senescence-associated $\beta$-galactosidase activity (Figures 6, F and G) and downregulation of $C d k n 1 a(p 21)$ in the CKP-N $\mathrm{N}^{\Delta \text { Panc }}$ tumor cells (Figure $6 \mathrm{H}$ ). Collectively, our data confirm that Wasl loss regulates pancreatic tumor cell senescence by both inducing the endosomal sequestration of the $\beta$-catenin destruction complex components GSK $3 \beta$ and YAP1 and by inducing mislocalization of p120ctn.

This study shows that N-WASP acts as an oncogene in the development of PDAC by regulating protein stability and subcellular localization of components of the p120ctn/ $\beta$-catenin signaling pathway.

\section{Discussion}

Kras $^{G 12 D}$ is the driver oncogenic mutation of PDAC (1). However, despite extensive research, the precise molecular mechanism involved in PDAC development and progression is not well understood. The effectors of oncogenic Kras include CDC42 and PIP2, both binding partners of N-WASP (encoded by Wasl) $(12-14,52)$, which is associated with poor PDAC prognosis (7). Originally, N-WASP was investigated in the pathogenesis of the human Wiskott-Aldrich syndrome, where it regulates immunity by interacting with histone methyltransferases (53). At a molecular level, N-WASP acts as an actin nucleation-factor (54); thus, it is not surprising that it is implicated in the regulation of cell migration and tumor metastasis (7-9, 11). More recently, nuclear N-WASP was reported as an inhibitor of p53-induced senescence in a model for skin tumor formation, further implicating an oncogenic role for N-WASP (10). However, 
Table 1. Morphometric quantification of WIP staining

\begin{tabular}{|c|c|c|c|c|c|c|c|}
\hline \multirow{2}{*}{$\begin{array}{l}\text { WIP staining } \\
\text { Genotype }\end{array}$} & \multicolumn{3}{|c|}{ Cytoplasmic } & \multicolumn{2}{|c|}{ Membranous } & \multirow[t]{2}{*}{ Nuclear } & \multirow[t]{2}{*}{ Stroma } \\
\hline & - & + & ++ & - & + & & \\
\hline CKP-N $N^{\Delta \text { Panc }}$ & $4 / 12$ & $7 / 12$ & $1 / 12$ & $9 / 10$ & $1 / 10$ & $4 / 12$ & $12 / 12$ \\
\hline CKP-N ${ }^{\text {het }}$ & $4 / 8$ & $2 / 8$ & $2 / 8$ & $1 / 8$ & $7 / 8$ & $8 / 8$ & $3 / 8$ \\
\hline CKP & $0 / 4$ & $3 / 4$ & $1 / 4$ & $0 / 4$ & $4 / 4$ & $4 / 4$ & $3 / 4$ \\
\hline
\end{tabular}

Morphometric quantification of the WIP staining described in Figure 4A. Representative pictures for absent (-), weak (+), or strong (++) cytoplasmic staining; membranous and nuclear or stromal staining are shown in Supplemental Figure 4B. The assessment of the staining was done in a blinded fashion.

N-WASP has also been shown to act as a tumor suppressor, for example, in early intestinal carcinogenesis, suggesting a tissue-specific function of this protein (55). Since N-WASP binds KRAS effectors and because actin polymerization is one of the pathways involved in PDAC development (15), we hypothesized that Wasl expression plays a tumor-promoting role in PDAC development.

Using established oncogenic Kras-based mouse models for PDAC, we show that the conditional deletion of Wasl delays PDAC development, leading to senescent tumors in the absence of the tumor suppressor Trp53, and abrogates tumor development in the CK mouse model, highlighting the oncogenic properties of Wasl in PDAC. In both mouse models, we observed increased lipomatosis, and RNAseq showed that Wisp2/Ccn 5 was among the significantly upregulated genes upon loss of Wasl. Wisp2 encodes a cytoplasmic protein downregulated in PDAC, which is involved in the suppression of cell proliferation, reviewed in ref. 56; Wisp2 also regulates adipogenic commitment (57). Both known functions of Wisp2 are consistent with the low proliferation rate and the increased fatty metaplasia observed after deletion of Wasl in the CKP
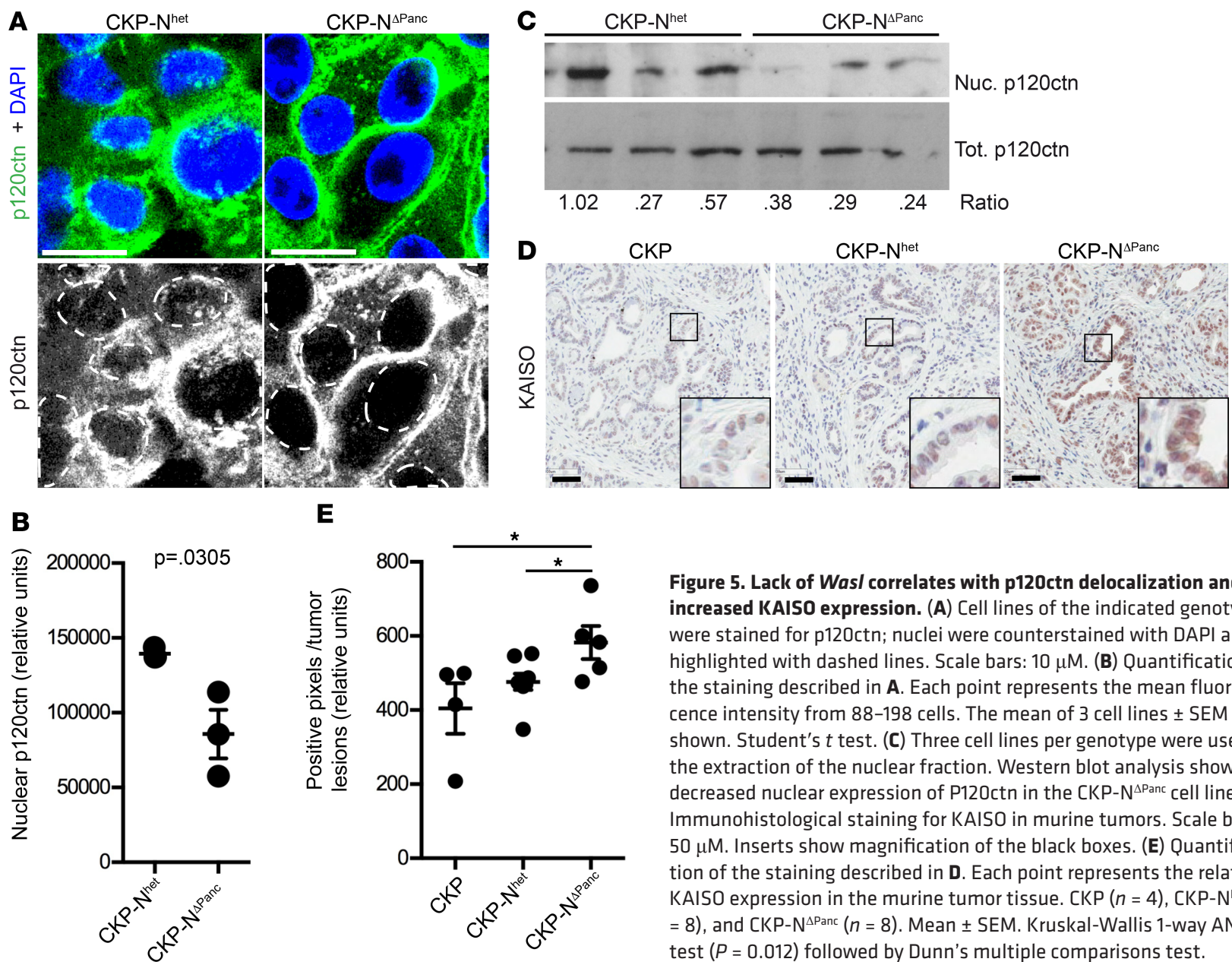

$\mathbf{E}$

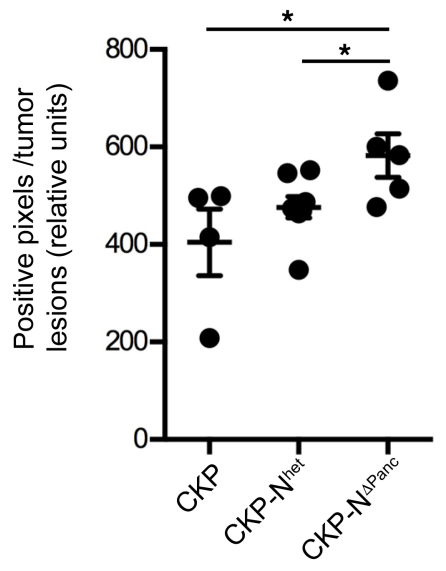

Figure 5. Lack of Wasl correlates with p120ctn delocalization and with increased KAISO expression. (A) Cell lines of the indicated genotype were stained for p120ctn; nuclei were counterstained with DAPI and are highlighted with dashed lines. Scale bars: $10 \mu \mathrm{M}$. (B) Quantification of the staining described in $\mathbf{A}$. Each point represents the mean fluorescence intensity from 88-198 cells. The mean of 3 cell lines \pm SEM is shown. Student's $t$ test. (C) Three cell lines per genotype were used for the extraction of the nuclear fraction. Western blot analysis shows a decreased nuclear expression of P120ctn in the CKP-N $\mathrm{N}^{\Delta \text { Panc }}$ cell lines. (D) Immunohistological staining for KAISO in murine tumors. Scale bars: $50 \mu \mathrm{M}$. Inserts show magnification of the black boxes. (E) Quantification of the staining described in $\mathbf{D}$. Each point represents the relative KAISO expression in the murine tumor tissue. CKP $(n=4)$, CKP-N ${ }^{\text {het }}(n$ $=8)$, and CKP-N $\mathrm{N}^{\triangle \mathrm{Panc}}(n=8)$. Mean $\pm \mathrm{SEM}$. Kruskal-Wallis 1-way ANOVA test $(P=0.012)$ followed by Dunn's multiple comparisons test. 
A
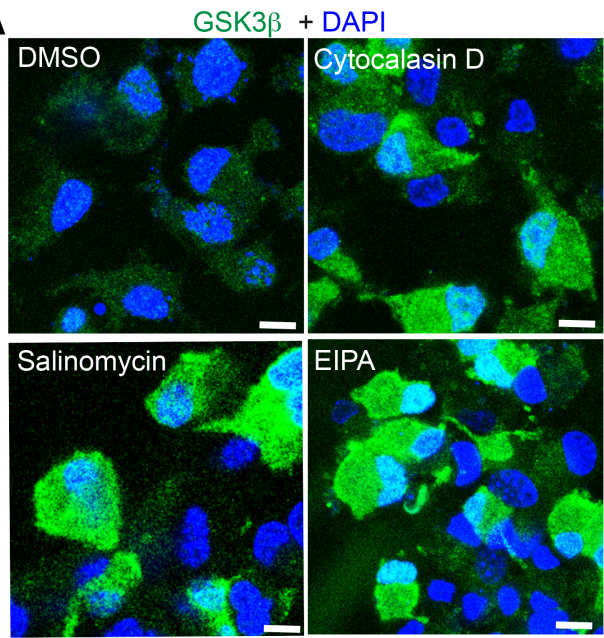

B

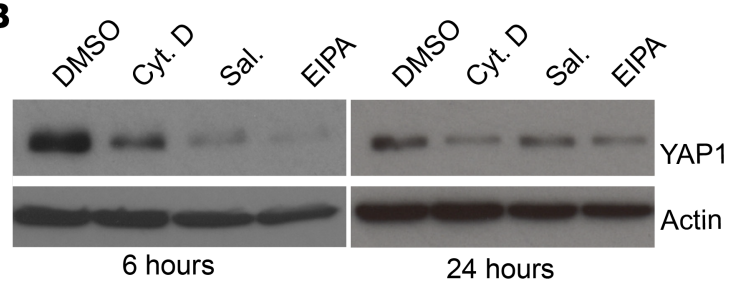

\section{C}

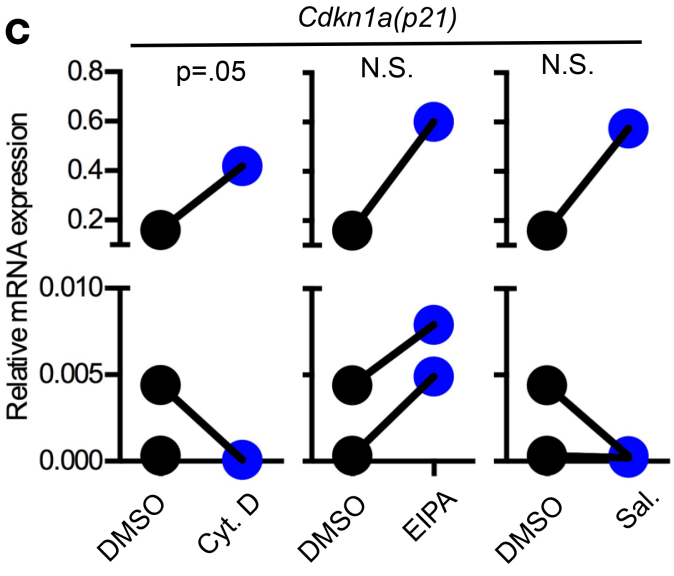

D

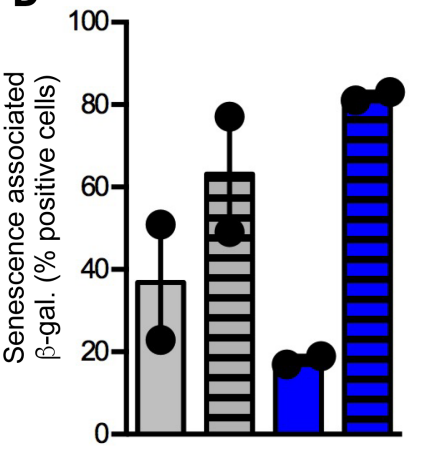

\section{DMSO \\ Sal. \\ $\square$ Weak staining \\ 目Strong staining}


$\mathbf{F}$

DMSO

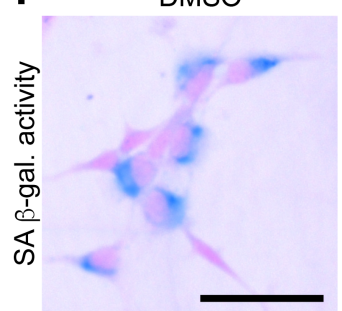

G

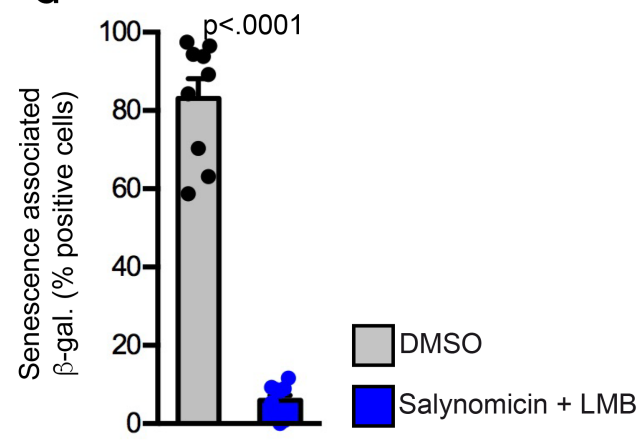

H

$C d k n 1 a(p 21)$

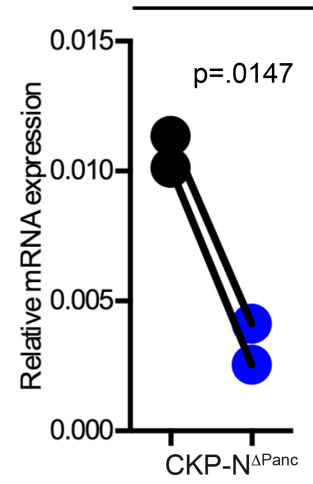

LMB $5 \mathrm{ng} / \mathrm{ml}$



Sal. + LMB

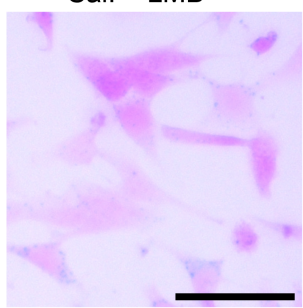

Salynomicin + LMB

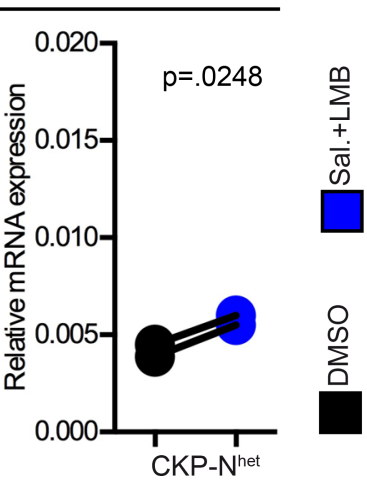

Figure 6. Combined salinomycin and LMB treatment of CKP-N $\mathbf{N}^{\Delta \mathrm{Panc}}$ tumor cells rescues the senescent phenotype. (A) CKP-N ${ }^{\Delta \mathrm{Panc}}$ cells were treated with Cytochalasin $\mathrm{D}(2 \mu \mathrm{M})$, salinomycin $(1 \mu \mathrm{M})$, or EIPA $(25 \mu \mathrm{M})$ for 48 hours; then, the cells were fixed and stained for CSK3 3 . Scale bars: $10 \mu \mathrm{M}$. (B) CKP-N $\mathrm{N}^{\Delta \mathrm{Panc}}$ cells were treated as described for $\mathbf{A}$, harvested, and analyzed by Western blot. (C) Three CKP-N ${ }^{\Delta \text { Panc }}$ cell lines were treated as described for $\mathbf{A}$ for 24 hours and harvested, and the relative Cdkn1a(p21) expression was analyzed by RT-PCR. Student's $t$ test. (D) CKP-N $\mathrm{N}^{\Delta \mathrm{Panc}}$ cells were grown on a thin layer of collagen and treated with salinomycin; then, the cells fixed stained for SA- $\beta$-galactosidase and quantified. The mean of 2 cell lines \pm SEM is shown. Student's $t$ test. 
(E) CKP-N $\mathrm{N}^{\Delta \text { Panc }}$ cells were treated with LMB $(5 \mathrm{ng} / \mathrm{mL})$ for 20 hours; then, the cells were fixed and stained for p120ctn. Scale bars: $50 \mu M$. (F) CKP-N $\mathrm{N}^{\Delta \mathrm{Panc}}$ cells were grown on a thin layer of collagen and sequentially treated with $1 \mathrm{M}$ salinomycin and $5 \mathrm{ng} / \mathrm{mL} L \mathrm{LB}$; then, the cells fixed stained for $S A-\beta$-galactosidase. A representative image is shown. Scale bars: $50 \mu \mathrm{M}$. (C) Quantification of the treatment described in F; each treatment was performed in triplicates. The mean of 3 cell lines \pm SEM is shown. Student's $t$ test. $(\mathbf{H})$ Cell lines were treated as described for panel $F$. The relative Cdkn1a(p21) expression of 2 cell lines is shown. Student's $t$ test.

mouse model. Notably, Wisp2 was slightly upregulated in 4-week-old CK-N ${ }^{\Delta \text { Panc }}$ mice, supporting an early function of Wisp2 upon loss of Wasl.

PDAC patients stratified for low WASL expression have improved survival and exhibit low expression of senescence markers, as well as downregulation of the Wnt/ $\beta$-catenin pathway; thus, mechanistically, we focused on the tumors developed by the CKP-N $\mathrm{N}^{\Delta \text { Panc }}$ mice, which showed similar molecular features to low $W A S L$-expressing human PDAC tumor tissues rather than investigating the $\mathrm{CK}-\mathrm{N}^{\Delta \mathrm{Panc}}$ mice, which do not develop tumors.

$\mathrm{CKP}-\mathrm{N}^{\Delta \mathrm{Panc}}$ mice developed slightly more mesenchymal tumors than control mice. This phenotype can be explained by the combined action of several factors, as described below. First, we observed high Kaiso expression in CKP-N ${ }^{\Delta P a n c}$ tumors. Since Kaiso is implicated in regulation of the mesenchymal phenotype and high Kaiso expression correlates with poor survival of many different tumor types (prostate, breast, pancreas; reviewed in ref. 48), this raises the possibility that Kaiso plays a role in Wasl-driven PDAC. Second, the CKP-N ${ }^{\triangle \text { Panc }}$ tumors have increased YAP1 expression, which is also involved in regulating the mesenchymal phenotype (58). Last but not least, our detection of increased inflammation (which is intimately connected to induction of epithelial-mesenchymal transition EMT; ref. 59) in CKP-N ${ }^{\Delta P a n c}$ and CK-N ${ }^{\Delta P a n c}$ mice further supports a Wasl mechanism involving EMT. Interestingly, although EMT is linked to worse PDAC survival outcomes (60), our CKP-N ${ }^{\Delta \text { Panc }}$ mice did not exhibit poor survival, most likely because there was no tumor metastasis. However, the improved survival of CKP-N ${ }^{\triangle P a n c}$ mice may also be explained by the low proliferating senescent tumors. We showed that loss of Wasl led to a cell-autonomous senescent phenotype in the tumors, consistent with strong upregulation of $C d k n 1 a(p 21)$ and increased SA- $\beta$-galactosidase activity that is currently the most accepted biomarker of senescence in vivo $(32,33)$. Cellular senescence has been described as a tumor-suppressive mechanism (36). However, senescent cells can modify the tumor microenvironment by acquisition of SASP, which can increase the number of inflammatory cells $(36,61)$, as evidenced by the GSEA signature for an inflammatory response in the CKP-N ${ }^{\Delta \text { Panc }}$ tumors.

Since no nuclear N-WASP was detected in CKP-N ${ }^{\Delta \text { Panc }}$ tumors, we focused on N-WASP interacting proteins (WIP and p120ctn) rather than on the deregulated genes $(12,13)$. N-WASP interacts with the actin cytoskeleton, which in turn regulates cell motility and is not linked to senescence $(12,13)$. N-WASP additionally binds WIP and p120ctn. WIP is required for N-WASP and YAP1 stabilization by sequestration of the $\beta$-catenin destruction complex $(12,17,40)$. YAP1 is involved in several signaling pathways, where it plays a different role depending on the upstream members (62). WIP is also involved in the regulation of endocytosis (63) and participates in several cellular processes, some of which are relevant in cancer and which may be dependent on different oncogenic stimuli $(17,64,65)$. We showed that $C K P-\mathrm{N}^{\Delta P a n c}$ tumors displayed a small amount of membranous WIP, which probably resulted in the increased rate of endocytosis that we observed in these tumors. In line with the role of WIP in the sequestration of destruction complex components, we observed vesicular staining of GSK3 $\beta$, as well as high YAP1 and phosphorylated $\beta$-catenin levels. Since inhibition of endocytosis induced cytosolic accumulation of GSK3 $\beta$ and decrease of YAP1 expression, we conclude that loss of N-WASP in our mouse model leads to sequestration of the destruction complex into vesicles. We recently showed that Wasl deletion impairs ADM by inhibiting fluid-phase endocytosis in pancreatic acinar cells (6). Juin and colleagues created a conditional mouse model with a mosaic deletion of Wasl and concomitant activation of oncogenic Kras and mutated p53 (Trp53 $\left.{ }^{R 172 H}\right)(\mathrm{KPCN}$ mice) (9). In this KPCN model, Wasl loss deregulated vesicular transport, reduced metastasis, and improved survival. These 2 studies in conjunction with our current study reveal a crucial role for N-WASP in PDAC development through regulation of vesicular trafficking.

The second N-WASP binding partner that we focused on is p120ctn. CKP-N $\triangle$ Panc tumors had less nuclear p120ctn, which is consistent with better prognosis of PDAC patients (18). p120ctn is also involved in the internalization of the GSK3 $\beta$-containing destruction complex into multivesicular bodies (66); thus, the delocalization p120ctn upon loss of Wasl likely affected the vesicular sequestration of destruction complex components. P120ctn shuttles to and from the nucleus, where it interacts with the transcription factor 
Kaiso, which is upregulated in CKP-N ${ }^{\Delta \text { Panc }}$ tumors $(43-45,48,67)$. Since we detected low p120ctn nuclear levels in the CKP-N $\mathrm{N}^{\Delta \text { Panc }}$ tumor cells, we reasoned that $\mathrm{p} 120 \mathrm{ctn}$ undergoes increased nucleocytoplasmic shuttling in our model. We were, however, only able to rescue the senescence-associated phenotype in CKP-N ${ }^{\Delta P a n c}$ tumor cells by inducing nuclear p120ctn translocation along with inhibition of endocytosis. In summary, our study implicates p120ctn nucleocytoplasmic shuttling and accumulation of destruction complex components in the senescence phenotype of CKP-N $\mathrm{N}^{\Delta \mathrm{Panc}}$ tumor cells and, by extension, suggest a role for N-WASP in these processes in PDAC tumor development.

Notably, lack of Wasl - in the context of oncogenic Kras only — led to an inflammatory phenotype at an early time point. Pancreatitis is considered an important factor that promotes both human and murine PDAC $(2,30,31)$. However, despite the inflammatory phenotype at 4 weeks of age, which completely regressed within 6 months, the $C K-N^{\Delta P a n c}$ mice did not develop tumors. Since injury of acinar cells can lead to fatty degeneration in pancreatitis (60), this is consistent with the CK-N ${ }^{\Delta P a n c}$ phenotype at endpoint. Similar to the CKP-N ${ }^{\triangle P a n c}$ mouse model, GSEA in CK-N ${ }^{\triangle P a n c}$ mice showed an increased IL-6 signaling signature, along with an INFG response. Thus, the $C K-\mathrm{N}^{\Delta \mathrm{Panc}}$ phenotype resembles some senescence features of the CKP-N ${ }^{\Delta \text { Panc }}$ mouse model. These findings are of potential interest to elucidate the role of inflammation in PDAC development and may be investigated in further studies.

In conclusion, Wasl is required for the suppression of Kras-induced senescence upon Trp53 loss $(27,35)$. Given the importance of senescence in PDAC (27), unraveling a connection between N-WASP, senescence, and the $120 \mathrm{ctn} / \beta$-catenin pathway may provide strategies for the treatment of pancreatic cancer.

\section{Methods}

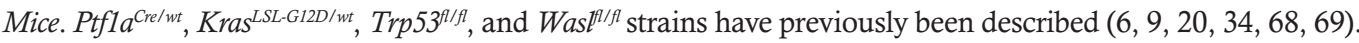

IHC and immunofluorescence staining. The primary antibodies used are listed in Supplemental Table 6. Immunoperoxidase staining was performed on 4\% PFA/PBS fixed paraffin-embedded tissue slides using the Vectastain-ABC-Elite and/or the ImmPRESS kit (Vector Laboratories) following manufacturer's instructions. For immunofluorescence staining, samples were fixed with 2\% PFA for 10 minutes and permeabilized with $0.1 \%$ Triton X-100 for 5 minutes. Slides were incubated overnight with primary antibodies (listed in Supplemental Table 6 ) at $4^{\circ} \mathrm{C}$ and were then incubated for 1 hour with secondary antibodies (Alexa dyes, 1:500, Invitrogen) at room temperature. Coverslips were mounted on tissue sections with VECTASHIELD HardSet Mounting Medium containing DAPI (Vector Laboratories) and examined using a Leica TCS_SP5 confocal microscope. In all experiments, samples were stained sequentially. To avoid bleed-through, single channels were scanned sequentially. Primary antibodies used are listed in the Supplemental Table 6.

Morphometric analysis. Slides from murine tissue were digitalized, and the regions of interest were quantified blindly using Aperio-ImageScope from Leica. Alternatively, several randomly chosen pictures were taken using a $10 \times$ objective from the stained slides, and positive staining was quantified blindly by different researchers.

Cell culture. Primary pancreatic tumor cells were derived from transgenic mouse tumors described above and maintained in DMEM media (Sigma-Aldrich, catalog D0819) supplemented with 10\% FCS (Thermo Fisher Scientific). Cells were incubated in a humidified incubator at $37^{\circ} \mathrm{C}$ and $5 \% \mathrm{CO}_{2}$. Where specified, the cell culture dishes were coated with a thin layer of collagen using rat tail collagen type I (Corning) according the manufacturer's instructions. For inhibition of endocytosis, the following inhibitors were used: salinomycin (MilliporeSigma, S6201), Cytochalasin D (Tocris, 1233/1), and EIPA (Tocris, 3378/10). LMB was purchased from Sigma-Aldrich.

Organoid isolation and maintenance. Tumor cells were isolated and cultured as previously described (19, $37,38,70,71)$. Briefly, after euthanizing the mice, the pancreas was quickly harvested and washed in PBS plus penicillin-streptomycin. The pancreas was then minced into small pieces and further digested with collagenase type IV (Sigma-Aldrich) for 1 hour. Additional digestion with Trypsin for 5 to 10 minutes was performed. Pancreatic cells were further washed and centrifuged at $200 \times g$ for 5 minutes at $4{ }^{\circ} \mathrm{C}$. The cells were mixed with Matrigel, and the mixture was plated (50 $\mu \mathrm{L}$ per well of a 24 -well plate) and allowed to solidify. After 20 minutes, when the Matrigel had solidified, complete culture media (37) was added to cells.

Immunoprecipitation and Western blots. Immunoprecipitation and Western blot analyses were performed as previously described $(6,44)$. Primary antibodies used are listed in Supplemental Table 6. Antibody binding was visualized using HRP-labeled secondary antibodies $(1: 10,000)$ (GE Healthcare, catalog NA935V, NA394V, and NA931V) and ECL reagent (GE Healthcare). 
Quantitative PCR. Total RNA extraction was performed according to manufacturer's instructions using QIAGEN RNeasy Plus Micro Kit (catalog 74034) for organoids and the NucleoSpin RNA kit (Machery-Nagel) for all other samples. RNA was reverse transcribed to cDNA using Super ScriptIII reverse transcriptase and oligo(dT) primers (Thermo Fisher Scientific) following manufacturer's instructions. The resultant cDNA was used as template for quantitative PCR (qPCR). RT-PCR was performed in duplicates, using the iTaq universal SYBR Green (Bio-Rad) according to manufacturer's instructions. Primers used are listed in Supplemental Table 7. The relative amount of each gene of interest was normalized to the housekeeping gene XS13 (an acidic ribosomal protein, which is constitutively expressed at the same level in normal, cancerous, and inflamed pancreas; ref. 24) using the $\triangle \triangle \mathrm{CT}$ method.

RNAseq and GSEA by Enrichr. RNA extraction was performed as described for qPCR. RNA concentration was fluorimetrically determined using the QuBit 3.0 system (Thermo Fisher Scientific). Bulk 3'-prime transcript end RNAseq (SCRB-Seq) libraries were prepared as described below. RNA was reverse transcribed using oligo(dT) primers decorated with sample barcodes, unique molecular identifiers (UMIs), and adapters (Integrated DNA Technologies). cDNA from all samples was pooled, and unincorporated primers were digested using Exonuclease I (New England Biolabs). The cDNA pool was then amplified with KAPA HiFi ReadyMix (KAPA Biosystems). To obtain sequencing libraries, $0.8 \mathrm{ng}$ of cDNA was fragmented, and $3^{\prime}$ ends were amplified with the Nextera XT Kit (Illumina) using a specific primer for the adapter on the $3^{\prime}$-end. The library was paired-end sequenced on a HiSeq1500 with 16 cycles for read 1 to decode sample barcodes and UMIs, and 51 cycles on read 2 into the cDNA fragment. Transcript and gene definitions were used according to the ENSEMBL annotation release 75. GSEA was performed using the web tool Enrichr, available at http://amp.pharm.mssm.edu/ Enrichr $(23,24)$. In addition, GSEA Software v4.0.3 (72) was used to calculate normalized enrichment scores (NES), false discovery rate (FDR) values, and $P$ values for gene sets in the Molecular signatures database (MSigDB) (73). The genes were preranked according to the fold-change and $P$ values

Endocytosis assay. A fluid-phase HRP uptake assay was used as described previously (6). Briefly, samples were incubated with $1 \mathrm{mg} / \mathrm{mL}$ HRP (MilliporeSigma) for 5 minutes. After pulse, chase, and extensive wash, cells were lysed (10 mM TRIS, $0.05 \%$ Triton X-100). The amount of internalized HRP was quantified by measuring the peroxidation of o-Dianisidine (MilliporeSigma) after addition of $\mathrm{H}_{2} \mathrm{O}_{2}$ at $405 \mathrm{~nm}$. The amount of internalized HRP was normalized to the total protein content measured by BCA protein assay (Pierce).

$S A-\beta$-galactosidase staining. SA- $\beta$-galactosidase staining was performed as previously described (33). Briefly, the fixed samples were incubated with a staining solution containing $20 \mathrm{mg} / \mathrm{mL}$ of X-Galactosidase (Roth) for $1-3$ days at $37^{\circ} \mathrm{C}$ and then mounted. Stained cells were examined with a Zeiss inverse microscope, and images were quantified blindly with ImageJ (NIH).

In silico patient survival. For stratification of PDAC patients, we used the UCSC Xena Platform (http:// xena.ucsc.edu) (74) with the TCGA Pancreatic Cancer (PAAD) data set filtered for primary tumors $(n=185)$.

Statistics. Statistical analyses were performed using the GraphPad Prism version 5.0a for Mac OS X. We applied unpaired, 2-tailed Student's $t$ test, Mann-Whitney $U$ test, the 2-way ANOVA, and the log-rank test; for multiple comparisons, we used the Kruskal-Wallis test followed by a Dunn's multiple comparisons test, as specified in the legends. A $P$ value less than 0.05 was considered significant.

Study approval. The present study in animals was reviewed and approved by the Government of Bavaria, Munich, Germany (AZ 5.2-1-54-2532-46-2014) and the IACUC of the Technical University of Munich. Experiments were conducted in accordance with the German Federal Animal Protection Laws.

\section{Author contributions}

AHS, ZD, JD, and K. Schulte performed and analyzed experiments. CLM designed experiments, analyzed data, and wrote the manuscript. AHS, ZD, JD, HE, RMS, and CLM discussed the manuscript. HKE, BBA, LR, JMD, and K. Steiger performed tissue staining and molecular analyses. ASAA performed experiment for the revision. ML planned and discussed senescence experiments. AHS, ZD, JD, BBA, LR, JMD, ML, ASAA, and MR edited the manuscript. RO, TE, and RR were in involved in RNAseq experiments and analysis. HE performed GSEA analyses MR, GVF, JTS, RMS, and CLM provided funding. 


\section{Acknowledgments}

We would like to thank D. Tuveson and C. Brakebusch for providing transgenic animals. We thank B.K. Bhatti for technical help. The results published here are, in part, based upon data generated by using the UCSC Xena Platform for cancer genomics data visualization and interpretation (http://xena.ucsc. edu). This project was supported by the Deutsche Forschungsgemeinschaft (LU1943/1 and LU1943/12, to CLM; SI1549/2-1 and SI1549/3-1 (KFO337), to JTS), the SFB1321 Modeling and Targeting Pancreatic Cancer (to RR, MR, GVF and RMS), the SFB824 (project C4 to JTS), and the German Cancer Aid Foundation (Deutsche Krebshilfe 111273 to MR and 70112505 to JTS).

Address correspondence to: Clara Lubeseder-Martellato, Klinikum rechts der Isar Technical University of Munich, Klinik und Poliklinik für Innere Medizin II, Ismaninger Strasse 2281675 München, Germany. Phone: 49.0.89.4140.6792; Email: clara.lubeseder-martellato@tum.de.

1. Almoguera C, Shibata D, Forrester K, Martin J, Arnheim N, Perucho M. Most human carcinomas of the exocrine pancreas contain mutant c-K-ras genes. Cell. 1988;53(4):549-554.

2. Bardeesy N, DePinho RA. Pancreatic cancer biology and genetics. Nat Rev Cancer. 2002;2(12):897-909.

3. Aichler M, et al. Origin of pancreatic ductal adenocarcinoma from atypical flat lesions: a comparative study in transgenic mice and human tissues. J Pathol. 2012;226(5):723-734.

4. Kopp JL, et al. Identification of Sox9-dependent acinar-to-ductal reprogramming as the principal mechanism for initiation of pancreatic ductal adenocarcinoma. Cancer Cell. 2012;22(6):737-750.

5. Means AL, et al. Pancreatic epithelial plasticity mediated by acinar cell transdifferentiation and generation of nestin-positive intermediates. Development. 2005;132(16):3767-3776.

6. Lubeseder-Martellato C, et al. Oncogenic KRas-induced Increase in Fluid-phase Endocytosis is Dependent on N-WASP and is Required for the Formation of Pancreatic Preneoplastic Lesions. EBioMedicine. 2017;15:90-99.

7. Guo JC, et al. N-wasp in pancreatic ductal adenocarcinoma: associations with perineural invasion and poor prognosis. World $J$ Surg. 2014;38(8):2126-2131.

8. Hou J, et al. N-WASP promotes invasion and migration of cervical cancer cells through regulating p38 MAPKs signaling pathway. Am J Transl Res. 2017;9(2):403-415.

9. Juin A, et al. N-WASP Control of LPAR1 Trafficking Establishes Response to Self-Generated LPA Gradients to Promote Pancreatic Cancer Cell Metastasis. Dev Cell. 2019;51(4):431-445.e7.

10. Li H, Petersen S, Garcia Mariscal A, Brakebusch C. Negative Regulation of p53-Induced Senescence by N-WASP Is Crucial for DMBA/TPA-Induced Skin Tumor Formation. Cancer Res. 2019;79(9):2167-2181.

11. Martin TA, Pereira G, Watkins G, Mansel RE, Jiang WG. N-WASP is a putative tumour suppressor in breast cancer cells, in vitro and in vivo, and is associated with clinical outcome in patients with breast cancer. Clin Exp Metastasis. 2008;25(2):97-108.

12. Alekhina O, Burstein E, Billadeau DD. Cellular functions of WASP family proteins at a glance. J Cell Sci. 2017;130(14):2235-2241.

13. Rohatgi R, Ho HY, Kirschner MW. Mechanism of N-WASP activation by CDC42 and phosphatidylinositol 4, 5-bisphosphate. J Cell Biol. 2000;150(6):1299-1310.

14. Eser S, Schnieke A, Schneider G, Saur D. Oncogenic KRAS signalling in pancreatic cancer. Br J Cancer. 2014;111(5):817-822.

15. Heid I, et al. Early requirement of Rac1 in a mouse model of pancreatic cancer. Gastroenterology. 2011;141(2):719-730.

16. Zhang W, et al. Downstream of mutant KRAS, the transcription regulator YAP is essential for neoplastic progression to pancreatic ductal adenocarcinoma. Sci Signal. 2014;7(324):ra42.

17. Gargini R, Escoll M, García E, García-Escudero R, Wandosell F, Antón IM. WIP Drives Tumor Progression through YAP/ TAZ-Dependent Autonomous Cell Growth. Cell Rep. 2016;17(8):1962-1977.

18. Mayerle J, et al. Up-regulation, nuclear import, and tumor growth stimulation of the adhesion protein p120 in pancreatic cancer. Gastroenterology. 2003;124(4):949-960.

19. Reichert M, Takano S, Heeg S, Bakir B, Botta GP, Rustgi AK. Isolation, culture and genetic manipulation of mouse pancreatic ductal cells. Nat Protoc. 2013;8(7):1354-1365.

20. Hingorani SR, et al. Preinvasive and invasive ductal pancreatic cancer and its early detection in the mouse. Cancer Cell. 2003;4(6):437-450.

21. Li Q, et al. Reg proteins promote acinar-to-ductal metaplasia and act as novel diagnostic and prognostic markers in pancreatic ductal adenocarcinoma. Oncotarget. 2016;7(47):77838-77853.

22. Morris JP, Cano DA, Sekine S, Wang SC, Hebrok M. Beta-catenin blocks Kras-dependent reprogramming of acini into pancreatic cancer precursor lesions in mice. J Clin Invest. 2010;120(2):508-520.

23. Chen EY, et al. Enrichr: interactive and collaborative HTML5 gene list enrichment analysis tool. BMC Bioinformatics. 2013;14:128.

24. Kuleshov MV, et al. Enrichr: a comprehensive gene set enrichment analysis web server 2016 update. Nucleic Acids Res. 2016;44(W1):W90-W97.

25. Wan $\mathrm{MH}$, et al. Review of experimental animal models of biliary acute pancreatitis and recent advances in basic research. $H P B$ (Oxford). 2012;14(2):73-81.

26. Kuilman T, et al. Oncogene-induced senescence relayed by an interleukin-dependent inflammatory network. Cell. 2008;133(6):1019-1031.

27. Porciuncula A, Hajdu C, David G. The Dual Role of Senescence in Pancreatic Ductal Adenocarcinoma. Adv Cancer Res. 2016;131:1-20. 
28. Diersch S, et al. Kras(G12D) induces EGFR-MYC cross signaling in murine primary pancreatic ductal epithelial cells. Oncogene. 2016;35(29):3880-3886.

29. Walz S, et al. Activation and repression by oncogenic MYC shape tumour-specific gene expression profiles. Nature. 2014;511(7510):483-487.

30. Carrière C, Young AL, Gunn JR, Longnecker DS, Korc M. Acute pancreatitis markedly accelerates pancreatic cancer progression in mice expressing oncogenic Kras. Biochem Biophys Res Commun. 2009;382(3):561-565.

31. Guerra C, et al. Chronic pancreatitis is essential for induction of pancreatic ductal adenocarcinoma by K-Ras oncogenes in adult mice. Cancer Cell. 2007;11(3):291-302.

32. Caldwell ME, et al. Cellular features of senescence during the evolution of human and murine ductal pancreatic cancer. Oncogene. 2012;31(12):1599-1608.

33. Lesina M, et al. RelA regulates CXCL1/CXCR2-dependent oncogene-induced senescence in murine Kras-driven pancreatic carcinogenesis. J Clin Invest. 2016;126(8):2919-2932.

34. Bardeesy N, et al. Both p16(Ink4a) and the p19(Arf)-p53 pathway constrain progression of pancreatic adenocarcinoma in the mouse. Proc Natl Acad Sci USA. 2006;103(15):5947-5952.

35. Morton JP, et al. Mutant p53 drives metastasis and overcomes growth arrest/senescence in pancreatic cancer. Proc Natl Acad Sci USA. 2010;107(1):246-251.

36. Coppé JP, Desprez PY, Krtolica A, Campisi J. The senescence-associated secretory phenotype: the dark side of tumor suppression. Annu Rev Pathol. 2010;5:99-118.

37. Boj SF, et al. Organoid models of human and mouse ductal pancreatic cancer. Cell. 2015;160(1-2):324-338.

38. Moreira L, Bakir B, Chatterji P, Dantes Z, Reichert M, Rustgi AK. Pancreas 3D Organoids: Current and Future Aspects as a Research Platform for Personalized Medicine in Pancreatic Cancer. Cell Mol Gastroenterol Hepatol. 2018;5(3):289-298.

39. Reichert M, et al. Regulation of Epithelial Plasticity Determines Metastatic Organotropism in Pancreatic Cancer. Dev Cell. 2018;45(6):696-711.e8.

40. Rajput C, et al. Neural Wiskott-Aldrich syndrome protein (N-WASP)-mediated p120-catenin interaction with Arp2-Actin complex stabilizes endothelial adherens junctions. J Biol Chem. 2013;288(6):4241-4250.

41. Ramesh N, Antón IM, Hartwig JH, Geha RS. WIP, a protein associated with wiskott-aldrich syndrome protein, induces actin polymerization and redistribution in lymphoid cells. Proc Natl Acad Sci USA. 1997;94(26):14671-14676.

42. Azzolin L, et al. YAP/TAZ incorporation in the $\beta$-catenin destruction complex orchestrates the Wnt response. Cell. 2014;158(1):157-170.

43. Daniel JM. Dancing in and out of the nucleus: p120(ctn) and the transcription factor Kaiso. Biochim Biophys Acta. 2007;1773(1):59-68.

44. Daniel JM, Reynolds AB. The catenin p120(ctn) interacts with Kaiso, a novel BTB/POZ domain zinc finger transcription factor. Mol Cell Biol. 1999;19(5):3614-3623.

45. Daniel JM, Spring CM, Crawford HC, Reynolds AB, Baig A. The p120(ctn)-binding partner Kaiso is a bi-modal DNA-binding protein that recognizes both a sequence-specific consensus and methylated CpG dinucleotides. Nucleic Acids Res. 2002;30(13):2911-2919.

46. Iioka H, Doerner SK, Tamai K. Kaiso is a bimodal modulator for Wnt/beta-catenin signaling. FEBS Lett. 2009;583(4):627-632.

47. Blattler A, Yao L, Wang Y, Ye Z, Jin VX, Farnham PJ. ZBTB33 binds unmethylated regions of the genome associated with actively expressed genes. Epigenetics Chromatin. 2013;6(1):13

48. Pierre CC, Hercules SM, Yates C, Daniel JM. Dancing from bottoms up - Roles of the POZ-ZF transcription factor Kaiso in Cancer. Biochim Biophys Acta Rev Cancer. 2019;1871(1):64-74.

49. van de Ven RA, Tenhagen M, Meuleman W, van Riel JJ, Schackmann RC, Derksen PW. Nuclear p120-catenin regulates the anoikis resistance of mouse lobular breast cancer cells through Kaiso-dependent Wnt11 expression. Dis Model Mech. $2015 ; 8(4): 373-384$.

50. Schenk M, Aykut B, Teske C, Giese NA, Weitz J, Welsch T. Salinomycin inhibits growth of pancreatic cancer and cancer cell migration by disruption of actin stress fiber integrity. Cancer Lett. 2015;358(2):161-169.

51. Pieters T, et al. p120 Catenin-Mediated Stabilization of E-Cadherin Is Essential for Primitive Endoderm Specification. PLoS Genet. 2016;12(8):e1006243

52. Zheng Y, et al. FAK phosphorylation by ERK primes ras-induced tyrosine dephosphorylation of FAK mediated by PIN1 and PTP-PEST. Mol Cell. 2009;35(1):11-25.

53. Taylor MD, et al. Nuclear role of WASp in the pathogenesis of dysregulated TH1 immunity in human Wiskott-Aldrich syndrome. Sci Transl Med. 2010;2(37):37ra44.

54. Benesch S, et al. N-WASP deficiency impairs EGF internalization and actin assembly at clathrin-coated pits. J Cell Sci. 2005;118(Pt 14):3103-3115.

55. Morris HT, et al. Loss of N-WASP drives early progression in an Apc model of intestinal tumourigenesis. $J$ Pathol. 2018;245(3):337-348.

56. Russo JW, Castellot JJ. CCN5: biology and pathophysiology. J Cell Commun Signal. 2010;4(3):119-130.

57. Grunberg JR, Hammarstedt A, Hedjazifar S, Smith U. The Novel Secreted Adipokine WNT1-inducible Signaling Pathway Protein 2 (WISP2) Is a Mesenchymal Cell Activator of Canonical WNT. J Biol Chem. 2014;289(10):6899-6907.

58. Shao DD, et al. KRAS and YAP1 converge to regulate EMT and tumor survival. Cell. 2014;158(1):171-184.

59. Khalafalla FG, Khan MW. Inflammation and Epithelial-Mesenchymal Transition in Pancreatic Ductal Adenocarcinoma: Fighting Against Multiple Opponents. Cancer Growth Metastasis. 2017;10:1179064417709287.

60. Wang S, Huang S, Sun YL. Epithelial-Mesenchymal Transition in Pancreatic Cancer: A Review. Biomed Res Int. 2017;2017:2646148

61. Pylayeva-Gupta Y, Lee KE, Hajdu CH, Miller G, Bar-Sagi D. Oncogenic Kras-induced GM-CSF production promotes the development of pancreatic neoplasia. Cancer Cell. 2012;21(6):836-847.

62. Piccolo S, Dupont S, Cordenonsi M. The biology of YAP/TAZ: hippo signaling and beyond. Physiol Rev. 2014;94(4):1287-1312.

63. Vaduva G, et al. The human WASP-interacting protein, WIP, activates the cell polarity pathway in yeast. J Biol Chem. 
1999;274(24):17103-17108.

64. García E, Machesky LM, Jones GE, Antón IM. WIP is necessary for matrix invasion by breast cancer cells. Eur J Cell Biol. 2014;93(10-12):413-423

65. Wu W, et al. Tp53 Mutation Inhibits Ubiquitination and Degradation of WISP1 via Down-Regulation of Siah1 in Pancreatic Carcinogenesis. Front Pharmacol. 2018;9:857.

66. Vinyoles M, et al. Multivesicular GSK3 sequestration upon Wnt signaling is controlled by p120-catenin/cadherin interaction with LRP5/6. Mol Cell. 2014;53(3):444-457.

67. Kelly KF, Otchere AA, Graham M, Daniel JM. Nuclear import of the BTB/POZ transcriptional regulator Kaiso. J Cell Sci. 2004;117(Pt 25):6143-6152.

68. Ardito CM, et al. EGF receptor is required for KRAS-induced pancreatic tumorigenesis. Cancer Cell. 2012;22(3):304-317.

69. Lommel S, Benesch S, Rottner K, Franz T, Wehland J, Kühn R. Actin pedestal formation by enteropathogenic Escherichia coli and intracellular motility of Shigella flexneri are abolished in N-WASP-defective cells. EMBO Rep. 2001;2(9):850-857.

70. Renz BW, et al. Cholinergic Signaling via Muscarinic Receptors Directly and Indirectly Suppresses Pancreatic Tumorigenesis and Cancer Stemness. Cancer Discov. 2018;8(11):1458-1473

71. Ruess DA, et al. Mutant KRAS-driven cancers depend on PTPN11/SHP2 phosphatase. Nat Med. 2018;24(7):954-960.

72. Subramanian A, et al. Gene Set Enrichment Analysis: A Knowledge-Based Approach for Interpreting Genome-Wide Expression Profiles. Proc Natl Acad Sci U S A. 2005;102(43):15545-15550.

73. Liberzon A, Subramanian A, Pinchback R, Thorvaldsdóttir H, Tamayo P, Mesirov JP. Molecular Signatures Database (MSigDB) 3.0. Bioinformatics. 2011;27(12):1739-1740.

74. Goldman M, Craft B, Kamath A, Brooks A, Zhu J, Haussler D. The UCSC Xena Platform for cancer genomics data visualization and interpretation. https://doi.org/10.1101/326470. Posted on bioRxiv September 26, 2019. 